Provided for non-commercial research and education use. Not for reproduction, distribution or commercial use.

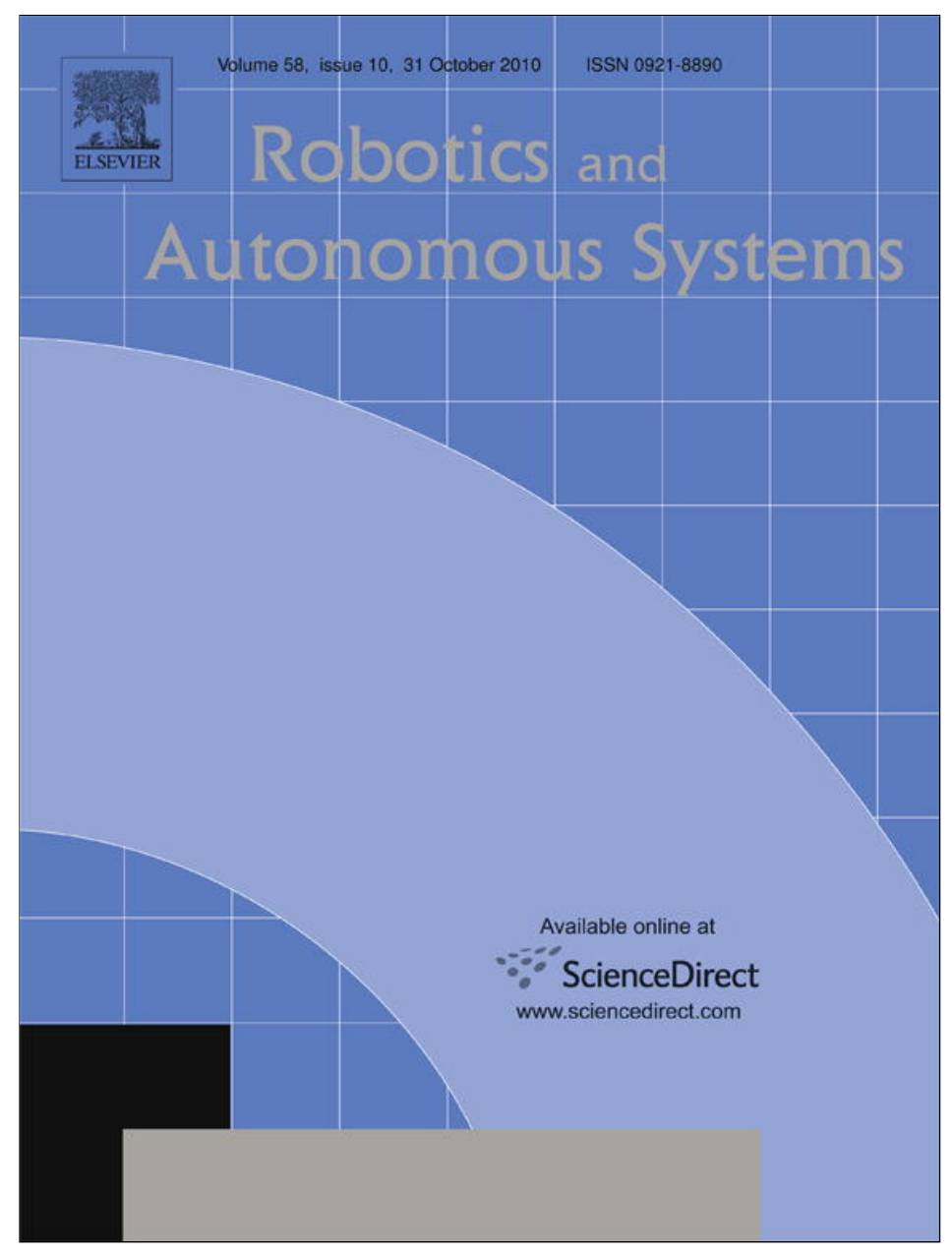

This article appeared in a journal published by Elsevier. The attached copy is furnished to the author for internal non-commercial research and education use, including for instruction at the authors institution and sharing with colleagues.

Other uses, including reproduction and distribution, or selling or licensing copies, or posting to personal, institutional or third party websites are prohibited.

In most cases authors are permitted to post their version of the article (e.g. in Word or Tex form) to their personal website or institutional repository. Authors requiring further information regarding Elsevier's archiving and manuscript policies are encouraged to visit:

http://www.elsevier.com/copyright 


\title{
A survey of Tactile Human-Robot Interactions
}

\author{
Brenna D. Argall*, Aude G. Billard \\ Learning Algorithms and Systems Laboratory (LASA), École Polytechnique Fédérale de Lausanne (EPFL), CH-1015 Lausanne, Switzerland
}

\section{A R T I C L E I N F O}

\section{Article history:}

Received 26 January 2010

Received in revised form

19 July 2010

Accepted 21 July 2010

Available online 1 August 2010

\section{Keywords:}

Human-Robot Interaction (HRI)

Tactile sensing

Physical human-robot contact

\begin{abstract}
A B S T R A C T
Robots come into physical contact with humans in both experimental and operational settings. Many potential factors motivate the detection of human contact, ranging from safe robot operation around humans, to robot behaviors that depend on human guidance. This article presents a review of current research within the field of Tactile Human-Robot Interactions (Tactile HRI), where physical contact from a human is detected by a robot during the execution or development of robot behaviors. Approaches are presented from two viewpoints: the types of physical interactions that occur between the human and robot, and the types of sensors used to detect these interactions. We contribute a structure for the categorization of Tactile HRI research within each viewpoint. Tactile sensing techniques are grouped into three categories, according to what covers the sensors: (i) a hard shell, (ii) a flexible substrate or (iii) no covering. Three categories of physical HRI likewise are identified, consisting of contact that (i) interferes with robot behavior execution, (ii) contributes to behavior execution and (iii) contributes to behavior development. We populate each category with the current literature, and furthermore identify the stateof-the-art within categories and promising areas for future research.
\end{abstract}

(C) 2010 Elsevier B.V. All rights reserved.

\section{Introduction}

Robots and humans come into physical contact under a variety of circumstances. For years robots have operated around humans within industrial and scientific settings, and their presence within the home and general society today becomes ever more common. During robot operation, physical contact with a human might be expected or unexpected, and enhance or interfere with the execution of a robot behavior.

Accordingly there are many motivations for detecting human touch within robot applications. This article provides an overview of current research within the field of Tactile Human-Robot Interactions (Tactile HRI): that is, of robot applications that detect and reason about, perhaps even depend on, the touch of a human. The field of tactile HRI represents the intersection of two independent research areas within robotics: the detection of tactile feedback, and interactions between humans and robots. In this review, we consider the field of tactile HRI from each of these perspectives.

\subsection{Tactile detection}

The area of tactile detection within robotics is a very broad field, with applications ranging from those which are strictly industrial

\footnotetext{
* Corresponding author. Tel.: +41 2169354 64; fax: +412169378 50 . E-mail addresses: brennadee.argall@epfl.ch (B.D. Argall), aude.billard@epfl.ch (A.G. Billard).

to those which involve social interactions with humans. Research within the area of tactile feedback aims to improve both the quality and interpretation of sensor data. Improvements in tactile sensing are measured according to the following criteria:

- Data quality. Evaluated according to detection sensitivity (range and resolution), noise (derived from the environment and other sensors) and physical robustness.

- Signal interpretation. Evaluated according to computational expense (time and storage) and measurement accuracy, and (occasionally) the sophistication of the extracted information.

For a review of tactile sensor devices and the algorithms that interpret their signals, we refer the reader to Nicholls and Lee [1] and Lee [2]; for a higher-level overview of advances in tactile robotics, to Howe [3]; for a more recent overview of grasping and manipulation approaches, and the hardware that such approaches typically employ, to Tegin and Wikander [4]; and for a current survey of tactile sensing for robotics, that additionally identifies design hints for sensor development, to Dahiya et al. [5]. In this review we will address only tactile sensing as used to detect human contact within HRI applications.

From the standpoint of tactile HRI detection, a variety of sensing approaches are taken in order to detect human touch. Approaches differ both in the sensor devices and data analysis techniques used for contact detection, as well as the types of touch that are able to be identified. Many applications build custom sensors able to perform an assortment of measurements by using multiple sensor devices with different detection targets. Interest in tactile sensing goes beyond the binary detection of human contact, and 
a variety of measurement data is extracted from these sensors. This data includes, but is not limited to: contact presence, location, area and duration; force magnitude, orientation and moment; vibration; temperature. Note however that at a minimum contact is always detected, and sensor devices which do not detect contact directly (e.g. potentiometers) are always paired with devices that do. The sensor devices employed for the detection of human touch include, but are not limited to: force/torque sensors, Force-SensingResistors (FSR), contact sensors, electric field sensors, capacitive sensing arrays, resistive sensing arrays, cameras, temperature sensors, potentiometers, photoreflectors, touchpads and straingauge sensors.

\subsection{Human-Robot Interaction}

The field of Human-Robot Interaction (HRI) is a research area that studies the interactions between robots and humans. The area is investigated for many reasons, such as to develop new techniques for knowledge transfer from human to robot, to design effective tools for human control of a robot, and in anticipation of the growing presence of robots within general society, to name a few. Moreover, the area is investigated from a variety of viewpoints, ranging from those which are human-centric, for example human-friendly interfaces for robot control, to those which are robot-centric, for example human guidance in the completion of a robot task. For the reader unfamiliar with HRI, we point to the following works for introductory information. An overview of HRI theory may be found in [6], with a focus on physical interactions being provided in [7]. Dautenhahn and Werry [8] discuss existing techniques, Steinfeld et al. [9] potential metrics, and Yanco and Drury [10] a proposed taxonomy for the analysis of HRI applications. This review will focus exclusively on HRI applications with a human touch element.

From the standpoint of HRI and robot behaviors, the detection of tactile interactions with a human is primarily motivated by at least one of the following considerations ${ }^{1}$ :

- Safe operation around humans. The robot possibly interacts with a human during behavior execution, perhaps unexpectedly (e.g. unintended collisions).

- A necessary element of behavior execution. The robot definitely, and necessarily, interacts with a human during behavior execution. The human might guide (e.g. indicate behavior selection) or be a partner in (e.g. human-robot team tasks) the execution, or the human-robot contact might be the entire point of the behavior (e.g. robot-assisted touch therapy).

- A necessary element for behavior development. The robot depends on tactile contact from a human while building, refining or adapting a behavior.

Approaches motivated by each of these considerations will be presented within this review.

\subsection{Outline}

This review presents the field of tactile HRI from two viewpoints. We contribute a structure for the categorization of techniques within each viewpoint, and furthermore place the current literature within this structure.

The first viewpoint, presented in Section 2, considers approaches within the context of tactile sensing. Three major tactile classes are identified, according to those sensors used for the detection of human touch within the recent literature. This section presents works at a technical level, providing sensor device details.

\footnotetext{
1 The majority of tactile HRI work to date however has been motivated by the first two considerations only.
}

The second viewpoint, presented in Section 3, considers approaches from the stance of physical human-robot interactions. Three major HRI classes are identified, according to the physical interactions seen in the literature to date. This section provides a presentation of works at a fairly high level.

A summary of the current state-of-the-art within the field of tactile HRI is then provided in Section 4, along with a discussion connecting tactile sensing techniques and HRI applications. Open areas for future research are identified, and in the final section we conclude.

\section{Tactile sensor feedback}

This section describes the mechanisms through which tactile feedback is detected and used within tactile HRI applications. We broadly organize the section according to details of the sensor setup used to detect the tactile feedback. Part of our discussion will be devoted to sensor skins, or mechanisms that combine multiple sensor devices with some sort of covering that takes on the shape of the robot body, with the result of continuous sensor coverage over the region covered by the skin. This covering might be a hard shell (Section 2.1) or flexible substrate (Section 2.2). Approaches that are not skin-based place individual or multiple sensors, often in the form of an array, on the robot body without any robot-shaped covering (Section 2.3).

Our organization of tactile sensing techniques takes motivation from the joint considerations of sensor covering and coverage, since both are important within the context of robot contact with a human. Regarding sensor covering, the form taken by (or the absence of) a covering for the sensors can limit the types of sensors that might be employed for contact detection, and furthermore heavily influences the (possibly negative) impact of the contact on both the human and the robot. Our categorization thus considers differences between hard and soft coverings over the sensors (i.e. hard skins vs. soft skins). Regarding sensor coverage, the extent (area) and resolution (density) of the sensor coverage plays a strong role in what types of human contact might be detected, and in which sorts of situations. Our categorization therefore further considers differences between individually placed sensors, sensor arrays and skins (i.e. skin alternatives vs. hard/soft skins). Other valid and meaningful categorizations of course are possible, for example based on what physical effects are detected by a given sensor (as summarized in Tables 2 and 3). We chose a categorization based on the distinctions of sensor coverage and covering because of the (HRI) focus of this survey, and the existence already within the literature of extensive technical reviews of tactile sensing technology (Section 1.1).

\subsection{Hard skins}

Hard, or bumper-based, skins consist of sensors covered by some sort of hard shell in the shape of the robot body. A variety of sensors have been implemented within such a design, for example force/torque sensors, FSRs, accelerometers and deformation sensors. Each panel of the shell frequently covers multiple sensors, whose data may be interpolated to provide measurement resolution at a finer scale than the size of a single shell panel.

\subsubsection{Analysis}

The hard shell affords bumper-based skins a ruggedness less available in soft skins. The trade-off is that the shell simultaneously restricts the type (e.g. no temperature or electric field) and resolution (e.g. much larger than $1 \mathrm{~cm}^{2}$ ) of the measurements that may be taken, or at least that have been taken as of this publication. Contact detection is not strictly limited to the locations of sensor 


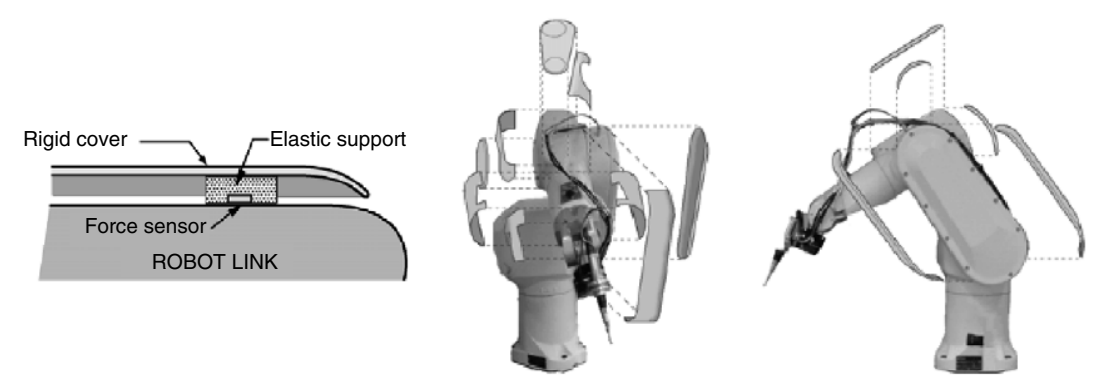

Fig. 1. The bump sensor (left) of Frigola et al. [12] covering an industrial robot arm (right).

devices on the robot body, since the shell is rigid and so the area surrounding the exact location of contact also is affected.

That the hard shell takes the shape of the robot body is one characteristic that distinguishes these skins from approaches (not addressed in this review, see Section 4.4) that attach a small number of individual contact sensors to the exterior of a robot body. Another distinguishing characteristic is that more information than just contact presence is detected; each panel of the shell is not simply an on/off switch.

\subsubsection{Applications}

The most common application for hard-shell skins is in the detection of, and recovery from, unintended collisions. Iwata et al. [11] cover the arms of their 52-DoF humanoid WENDY in 6 -axis force/torque sensors ( 6 per arm) and FSR sensors, followed by a hard plastic covering in the shape of the robot arm. This skin detects contact presence and position, as well as force magnitude, orientation and duration, and is able to detect multiple contacts simultaneously at several locations.

A skin of rigid bump sensors developed by Frigola et al. [12] covers all movable links of an industrial robot arm, with the stated aim of exploiting both the robustness of hard-shell skins and the detection sensitivity of soft array-based skins. The skin consists of deformation sensors in rubber (3-4 per panel), placed under a rigid metal sheet covering (Fig. 1). ${ }^{2}$ The work of Koo et al. [13] aims to develop a robot system that identifies and classifies different types of human touch without specialized sensors, which the authors consider to include covering the (typically hard) surface of a robot in a soft skin. Their skin is composed of two contact sensors and an accelerometer attached to the inside of a hard plastic cover.

\subsection{Soft skins}

Soft skins consist of sensors set upon, embedded within, or covered by some sort of flexible substrate. A variety of sensors have been implemented within such a design - for example potentiometers, FSRs, capacitance sensors, temperature sensors, electric field sensors, and photoreflectors - and in many cases multiple different sensor types are incorporated into a single skin. The flexible substrate enables the skin to cover a variety of body shapes, including curved surfaces. Substrate flexibility furthermore allows for very high spatial resolution.

\subsubsection{Analysis}

For the majority of soft skins, the tactile sensors are organized into arrays. In contrast to sensor arrays not paired with a flexible substrate (see Section 2.3.2), soft skins are able to perform tactile detection in areas not covered directly by a tactile sensing, since

\footnotetext{
2 Unless otherwise noted, all images within this review are taken from the cited publications with which they are presented.
}

the deformation of the flexible substrate propagates the tactile signal to the sensor, allowing for interpolation. In other cases, the substrate itself is the sensing device. For sensors embedded under or within the substrate, the covering additionally affords some level of protection. From an HRI perspective, a soft skin also allows for a soft contact sensation for the human and furthermore can feel similar to human skin, an important quality for robots aiming to have highly anthropomorphic characteristics.

The flexible substrate and sensor-network flavor of soft arraybased skins affords them sophisticated sensing capabilities less present with hard skins. The absence of a hard physical barrier between the sensor and contact object lifts the restriction of sensing only information related to contact presence and force (e.g. position, magnitude, orientation, duration), and a host of additional measurements may be taken, for example temperature, kinesthetic information and changes in electric field. As a tradeoff, the absence of this hard barrier technically makes the skin sensors more susceptible to damage, though admittedly little if any is reported within the literature. Furthermore, with soft skins the complication of self-touches at the robot joints can become an issue. Self-touches are a natural consequence of continuous coverage by a flexible sensor, whose deformability makes coverage possible in areas otherwise inaccessible with other technologies, for example that incorporate a hard-shell cover.

The spatial resolution of array-based skins has become impressively small, on the order of millimeter resolution within the most recent literature, and thus approaching the resolution of the human skin. From the robot's perspective, finer spatial resolution in general is considered to improve sensing capabilities. From the human's perspective, increasingly fine spatial resolution might eventually degrade his ability to provide feedback when touch is intended as a form of human-robot communication, since knowing exactly what is detected can become more difficult with increasing signal complexity.

\subsubsection{Applications}

In contrast to hard-shell skins, the most common application for soft skins is within the context of tactile feedback that contributes to behavior execution. An exception however is later work with the WENDY robot, which replaces the hard skin with a soft arraybased skin (Fig. 2); specifically, flexible FSR sensors cover the robot shoulder and arms ( $2 \mathrm{~cm}$ spatial resolution), and are further covered by an artificial skin made of elastomer-type silicone ( $3 \mathrm{~mm}$ thick) [14].

A succession of skin sensors are developed for the Robovie series of robots. The earliest consists of patches of pressure-sensitive conductivity rubber, 10 of which are placed on the Robovie-II [16]. An intermediate step covers Robovie's humanoid top entirely with this rubber, and information from these sensors combines with that of 10 tactile sensors located around the mobility base of the robot [17]. The final, full skin sensor is composed of tactile sensing elements embedded in a soft substrate, and consists of four layers: thin silicone rubber, a film-type piezoelectric sensing 


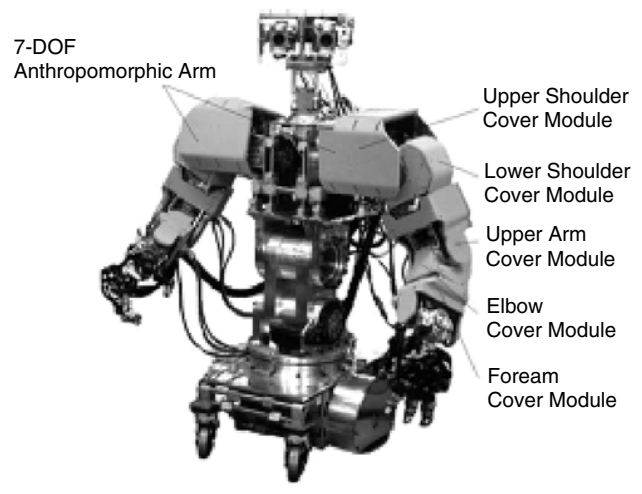

Fig. 2. The WENDY robot [14].

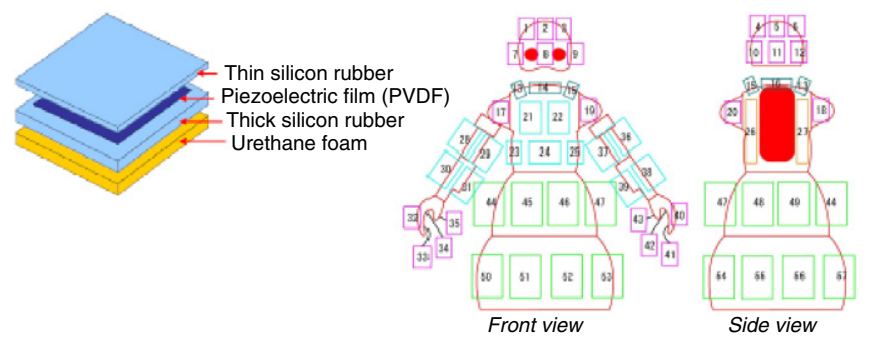

Fig. 3. The flexible skin sensor (left) covering the body of Robovie-IV (right). Marked locations on the body indicate PVDF positions, while the foam and rubber layers cover the entire body [15].
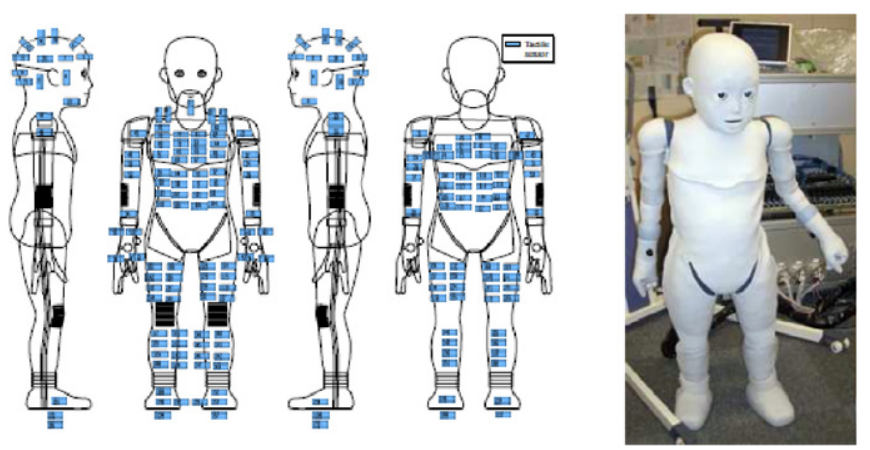

Fig. 4. Tactile sensor placement (left) under the skin covering the full body of the $C B^{2}$ robot (right) [20].

element (PVDF), thick silicone rubber, and urethane foam (Fig. 3). Two versions of Robovie-IIS are covered entirely in the silicone skin, with the first version having 48 sensing elements and the second 276 [18]. The skin also covers the body of Robovie-IV with 56 sensing elements [15], and later the body of Robovie-IIF with a total of 276 [19].

Work within this lab $^{3}$ also covers highly anthropomorphic humanoid robots in this silicone skin, in addition to the various versions of Robovie. The tactile skin covers the left arm of the prototype android ${ }^{4}$ Repliee $R 1$ [21]. The 42-DoF android robot Repliee Q2 [22] has its hands, lower arms and face covered in the silicone skin, for a total of 42 tactile sensors including those under the clothes (which cover all areas not covered by the skin). The

\footnotetext{
3 The Intelligent Robotics Laboratory, Department of Adaptive Machine Systems, Osaka University.

4 The authors define an android as a robot whose behavior and appearance is highly anthropomorphized; by contrast, a humanoid is a robot equipped with devices (e.g. head, eyes or hands) that make its appearance more human-like and enable analogous human movements and gestures.
}

geminoid $^{5}$ robot HI-1 [23] takes on similar specifications to the Repliee Q2. Finally, the robot $C B^{2}[20]$ is a child-sized android that, in contrast to the Repliee Q2 and geminoid HI-1, has its body covered entirely in the silicone skin (Fig. 4). This continuous skin coverage introduces a new challenge of self-touches at all joints, which the authors plan to address by introducing a temporal component to behavior execution and combining this with knowledge of self-movement.

The skin of Mukai et al. [24] embeds a tiling of FUJIKURA piezoresistive semiconductor pressure sensors in an elastic body. Skin specifications target the sensing capabilities of a human when carrying another human in her arms $(0-20 \mathrm{~mm}$ spatial resolution and $0.434-R 4.46 \frac{\mathrm{kg}}{\mathrm{cm}^{2}}$ pressure range, similar respectively to the human palm and $20 \times 20 \mathrm{~cm}^{2}$ of arm contact while holding a $60 \mathrm{~kg}$ human). The skin covers the arms and chest of the $158 \mathrm{~cm}$ tall humanoid RI-MAN (Fig. 5, left). In Yoshikai et al. [25], the entire body of the small 22-DoF humanoid macra is covered in a skin that embeds 3-axis force sensors (49 in total) in a soft urethane foam (Fig. 5, right).

A modular skin of pressure-sensitive elements developed by Ohmura et al. [26] is able to be folded and cut, with sensor coverage being adjustable via the addition/deletion of modules and sensor density adjustable by folding the bandlike bendable substrate on which the sensor sits. The pressuresensitive elements communicate via serial bus and each element consists of a photo-reflector $(3.2 \times 1.1 \times 1.7 \mathrm{~mm})$ covered by urethane foam (skin thickness $5 \mathrm{~mm}$ ). Foam deformation causes the detected light to scatter, and foam thickness controls the dynamic range and sensitivity of the sensor. The entire body of a 46-DoF humanoid is covered in this skin [27], with a total of 1864 sensing elements (Fig. 6).

We conclude with a set of applications that place a soft sensor skin under synthetic fur on robots with a stuffed-animal appearance. A skin of sensors is placed below the fur of the Paro robot [28], where tactile sensors formed of a dielectric material are able to sense force magnitude and location [29]. The Sensitive Skin of Stiehl et al. [30] embeds sensors within silicone, and is able to sense touch (electric fields and force), temperature (thermistors), kinesthetic information (potentiometers) and pain (a sharp reading in any sensor). The skin additionally is able to detect very soft touches (using electric field) and whether the source of a touch is a human or an object (based on temperature and electric field tuning). A single arm of the Huggable robot (Fig. 7) is equipped with 220 force, 80 temperature and 9 electric field sensors, with a net total over the whole body of more that 1000 force, 400 temperature and 9 electric field sensors.

\subsection{Alternatives to skin-based approaches}

A final set of approaches do not cover the tactile sensors with any sort of covering or skin. Some infer tactile information from readings taken off sensors located within the robot, commonly referred to as Intrinsic Contact Sensing, though most place sensors on the robot surface, either individually or organized into arrays.

Alternatives to skin-based approaches accordingly include force/torque sensors within a robot joint, often the "wrist" of a manipulator arm. Approaches also place surface sensors - such as pressure-sensitive conductivity rubber, FSRs or commercial tactile sensing pads - individually on the robot body. Other works instead organize surface sensors into arrays that either build on existing sensor technology, such as commercial FSRs, or incorporate novel tactile sensors, for example a fabric made from electrically conductive string.

\footnotetext{
5 The authors introduce a geminoid as a robot that functions as a duplicate of an existing person, to the extent that the appearances of the robot and human match and the robot is teleoperated by the human it duplicates.
} 

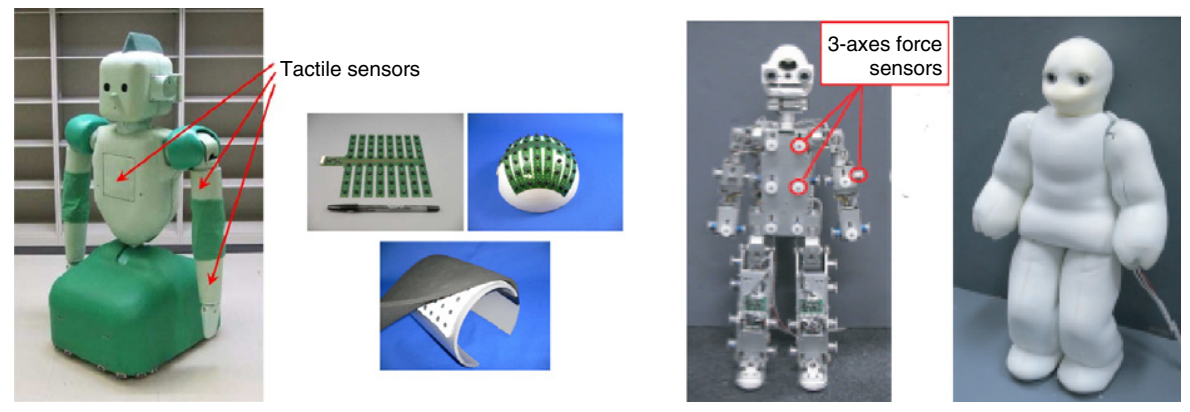

Fig. 5. The humanoids RI-MAN (left) and macra (right), each covered in an array of sensing elements embedded in a soft substrate [24,25].
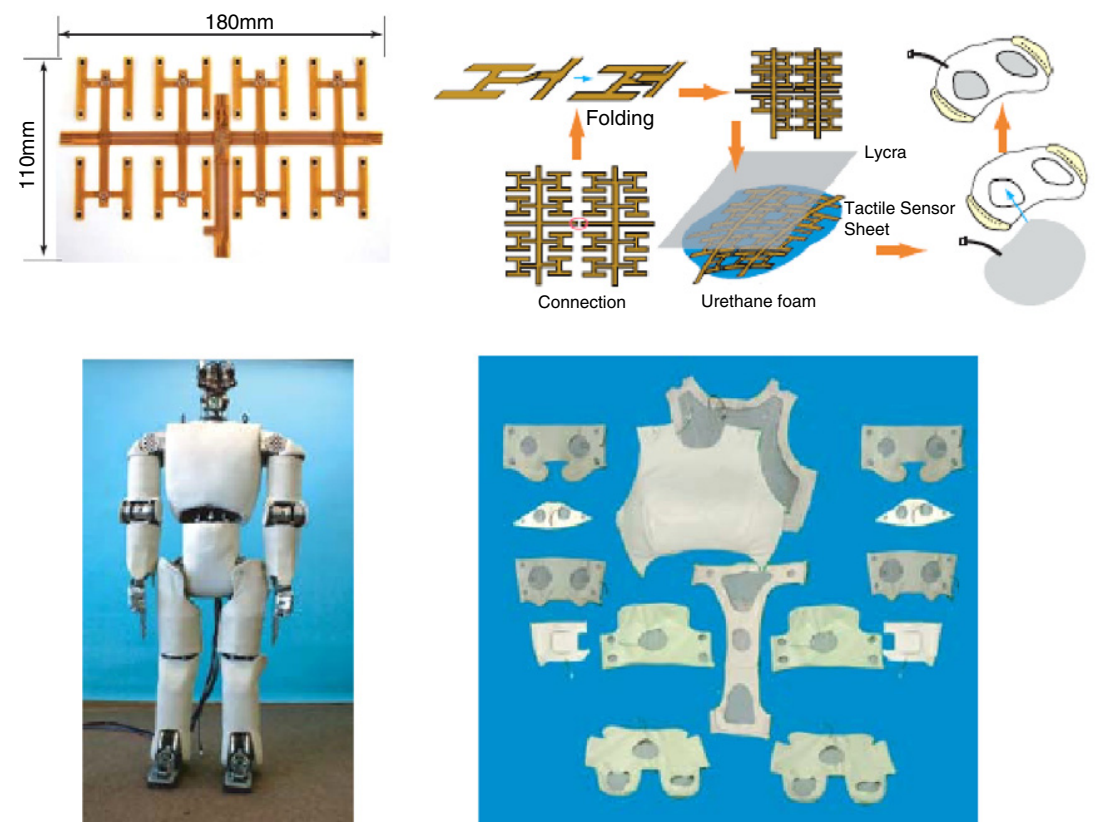

Fig. 6. Flexible tactile sensor (top) able to be folded and cut, that covers the entire humanoid body (bottom) of Ohmura and Kuniyoshi [27].
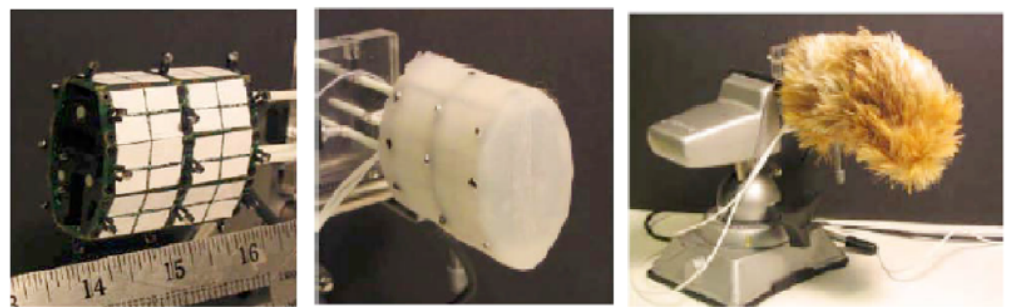

Fig. 7. Multi-modal tactile sensors (left) embedded in silicone (center) and covered in fur (right) for the Huggable robot [30].

\subsubsection{Analysis}

An advantage to using internal joint sensors is that the sensor technology is fairly non-specialized and well-established, having been applied to non-HRI domains like industrial environments for years. The disadvantage is that the tactile information able to be extracted from such data is fairly limited, for example the spatial resolution is typically quite low and often contact location is not able to be inferred at all.

Sensors located on the surface of the robot body have the advantage of offering a wide variety of sensor readings, and at a potentially high resolution. In comparison to skin-based approaches, the absence of a covering means that the sensors are left unprotected (which may or may not be an issue, depending on the sensor). Also, the acquisition of sensor readings at locations where no sensor is physically located, i.e. ubiquitous sensing, is generally not possible without some sort of continuous covering.
The gains from ubiquitous sensing decrease, however, as the spatial resolution of sensor arrays increases. The absence of the covering requirement also reduces, possibly even eliminates, the need for specialized hardware.

\subsubsection{Applications}

Here we begin with a brief discussion of tactile sensing inferred from sensors located within the robot body; that is, of force/torque sensing within a robot joint. ${ }^{6}$ We then proceed to the bulk of approaches under the topic of skin alternatives, that

\footnotetext{
6 For clarity, we emphasize that much of the research in tactile sensing, particularly in force/torque sensing, has been performed outside the realm of tactile HRI, and furthermore that within the realm, most applications fall beyond the scope of this review (see Section 4.4).
} 

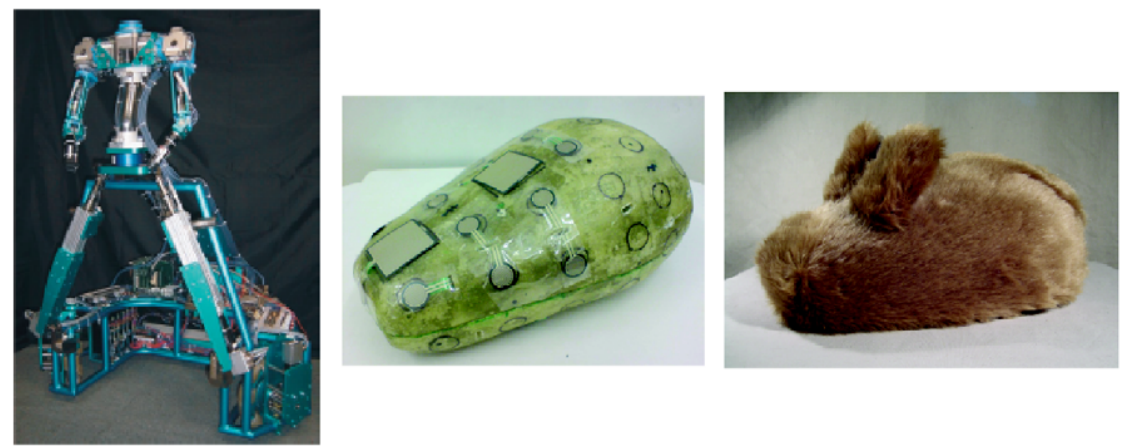

Fig. 8. The Partner Ballroom Dancing Robot (PBDR) (left, [31]). Sensor array (middle) covered by synthetic fur (right) for the robotic creature [32].

detect touch through sensors located on the surface of the robot body.

Industrial robot arms are a prominent application for joint/ torque sensing. Nudges to the end-effector of a PUMA arm are detected for behavior selection through a wrist force/torque sensor, or alternatively from a remote track ball, in the work of Voyles and Kholsa [33]. Both the location and strength of human touch is sensed via joint-torque sensors within all joints of an industrial-application lightweight robot arm in Grunwald et al. [34]. An exception to the industrial arm generalization is the Mobile Smart Dance Robot (Ms DanceR) [35], for which a single force/torque sensor located between the humanoid upper body and omnidirectional mobile base of the lower robot body detects planar rotational motion (force along $x, y$ and moment about $\theta$ ). The more recent Partner Ballroom Dance Robot (PBDR) [31] builds on the technology of the Ms DanceR, with an actuated waist between the humanoid top and omnidirectional base (Fig. 8, left).

We cover only a handful of approaches that place individual sensors on the body of the robot (see Section 4.4). Contact sensors on the robot body perform pinch detection at all major joints like the armpit and back of the knee on the entertainment robot SDR-4X II (QRIO) [36]. In an earlier version of Shibata and colleagues' seal robot Paro, contact sensors are placed at the ends of 2 whiskers, and 10 pressure sensors within balloons beneath artificial fur are able to detect pushing, patting and stroking [37]. The balloon pressure sensor was first used with their earlier "dog" robot, to replace hard tactile sensors that were displeasing to the humans interacting with the robot [38]. Shibata and colleagues also present a cat robot able to detect stroking, hitting and touching through piezoelectric force sensors along its back and on its head, as well as microswitches on its chin and cheeks [37]. The robotic creature robot of Yohanan and MacLean [32] detects human touch through 60 FSR sensors placed under the creature's fur (Fig. 8, right).

Early applications with sensor arrays on the robot body demonstrate impressive spatial resolution, though contact detection is binary and consequently quite limited in comparison to the variety of measurements gathered with more recent sensor array applications within soft skins. For example, a gridded pressure-sensitive ink sheet (1936 sensing elements in $16.5 \times 16.5 \mathrm{~cm}$ ) was developed by Naya et al. [39]. A sensor suit developed by Inaba et al. [40] from electrically conductive fabric and string covered the body of a 37-DoF humanoid robot, and data from 192 binary switch sensing regions $(10 \times 5 \mathrm{~cm}$ resolution on the leg, $5 \times 5 \mathrm{~cm}$ on the upper arm) was projected onto stereo vision images of the robot.

More recent work by Schmidt et al. [41] develops a novel sensor consisting of an array of 16 capacitive sensors that couple to the object being grasped via small brushes of fibers for dynamic sensing; thus providing two types of dynamical tactile information - speed and vibration - as a parallel to the two types detected by human skin (Fig. 9). This array furthermore is paired with two foil-based piezoresistive force sensors for static sensing. The arraybased work of Wosch and Feiten [42] covers an 8-DoF manipulator

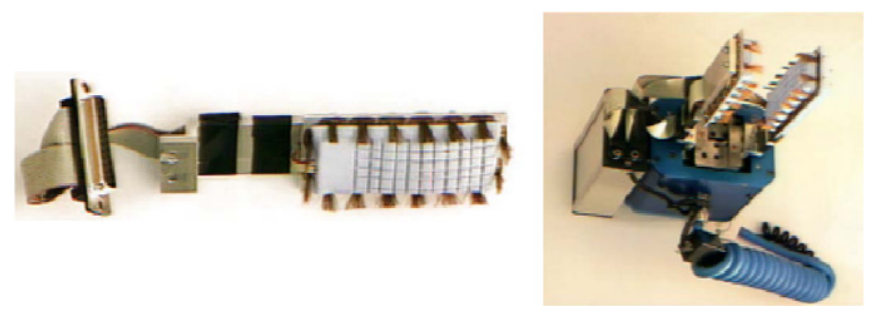

Fig. 9. Sensor for detecting static and dynamic tactile contact [41].

arm in 45 conductive foam force sensors. Schmid et al. [43] detect contact regions via pixel signature through an iGesture Pad, or alternatively a custom resistive pressure sensor array, placed on the robot arm. Multiple relational parameters are extracted from a single finger stroke (e.g. direction, distance, speed). Finger swipes and taps are detected by 4 Ergonomic Touchpads that encircle the wrist of the 53-DoF iCub humanoid in the work of Argall et al. [44]. Minato et al. [45] place 90 pressure sensors over the body of a small 22-DoF humanoid, the BabyBot (Fig. 10). The novel pressure sensors consist of a small hard plastic plate with a spring-like attachment to the robot body, which covers a photo emitter and interrupter pair able to detect changes in pressure.

\subsection{Summary}

From the sensor viewpoint, we have identified three primary tactile HRI topic areas: hard skins, soft skins and alternatives to skin-based approaches. A summary of the different forms taken by tactile detection within the current literature is provided in Tables 2 and 3 (What is detected).

The majority of recent work in tactile HRI, as well as its most sophisticated applications, falls within the category of soft skins. The state-of-the-art in soft skins has high-spatial resolution $[27,28,30]$, is able to take a variety of data measurements by incorporating multiple sensor devices into the skin substrate [30] and has continuous full body coverage $[15,20]$. Also worthwhile to note are dense, full-body, (not skin-based) sensor arrays [45], and approaches that take explicit steps in the direction of reproducing the sensing capabilities in the human skin [41].

\section{Physical human-robot interactions}

This section presents the different forms taken by physical human-robot interactions within the current tactile HRI literature. We classify the majority of these HRI forms into three categories. The first are physical interactions that interfere with the execution of a robot behavior (Section 3.1). The most common motivation for detecting such interactions is to enable safe robot operation in the presence of humans. The second category are interactions that are an intended part of robot behavior execution (Section 3.2). 

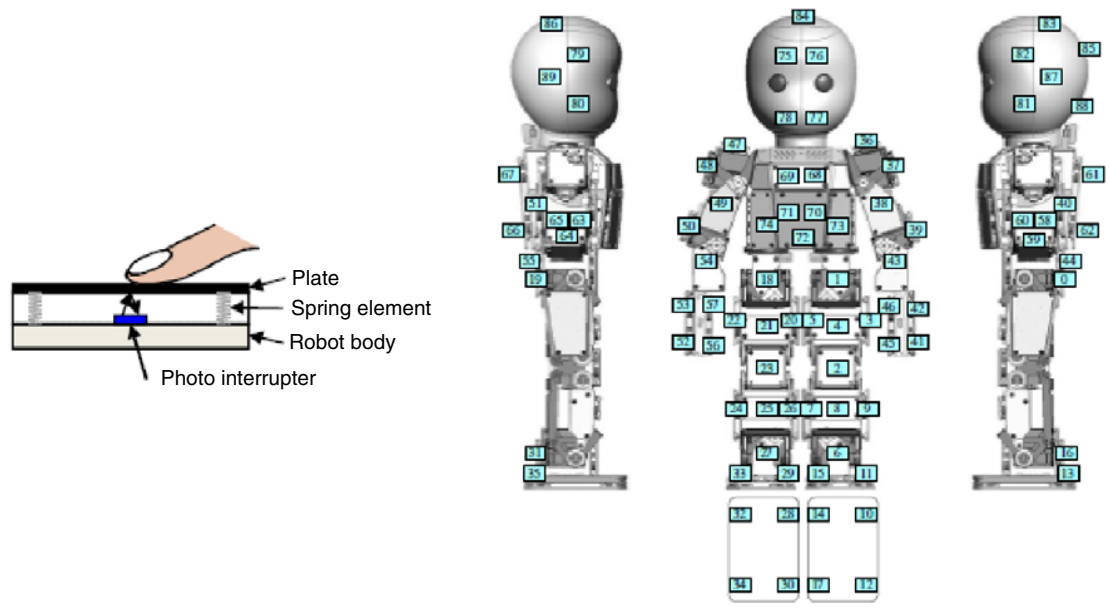

Fig. 10. Novel pressure sensor (left) and sensor placement (right) on the BabyBot humanoid [45].

Contact in this category is interpreted as an explicit means of communication between the human and robot, and its detection is necessary to facilitate human-robot communication during behavior execution. The third category includes interactions that build and refine robot behaviors (Section 3.3). The motivation here is to use the detected contact to produce adaptive and compliant robot behaviors, as a flexible alternative to hand design.

The approaches presented in this section generally take one of two perspectives as motivation for their work with physical HRI. The first is human-centric, where the detection of touch is intended to keep humans safe when interacting with robots. The second is robot-centric, where human touch is a tool through which robot behaviors are executed, built or adapted.

\subsection{Interactions that interfere with robot behavior execution}

We first discuss physical interactions between a human and robot that interfere with the execution of a task or behavior by the robot. These tactile interactions are not an intended or necessary part of the task the robot is executing. In some cases, however, the interaction might be represented within the robot behavior, so that the robot may intelligently recover from the disturbance. Unexpected human-robot contact is acknowledged to be an unavoidable occurrence as robots become more commonplace within human environments, especially given that human environments are frequently very dynamic and unpredictable. Physical contact between a human and robot could be quite dangerous for the human, and potentially damaging also to the robot. A primary goal of detecting human-robot contact that interferes with robot task execution, therefore, is to enable safe robot operation around humans.

The majority of approaches focus on the robot reactively responding to physical contact. An 8-DoF manipulator arm responds evasively to touch for human-obstacle avoidance during movement execution [42]. The redundant degrees of freedom in an industrial arm are exploited to maintain end effector position under external disturbances caused by a human, for evasive and safety measures $[12,34]$. The small entertainment humanoid SDR$4 X$ II performs pinch detection at major joints to avoid trapping or injuring the detected (possibly human) object. This robot also detects being lifted via a touch sensor on the back handle and force sensors in the feet, and in response makes the joints in its arms and legs compliant, for easier and safer handling by a human [36].

The research of Iwata and colleagues takes a very active approach to interference interactions, by predicting the effects of physical human-robot contact and encoding an appropriate response within the robot behavior. Early work introduced the term Physical InterFerence (PIF), which by their definition refers to all contact situations with a human from the robot's point of view [11]. One important consideration of this work is that task un-fulfillment might also injure the human, in addition to the physical contact with the robot, for example if a collision furthermore means that hot coffee is spilled. The authors therefore focus on the development of PIF Adapting Behaviors, where the robot understands the effects of its participation in PIF situations, both on the human and on its own task performance (Fig. 11). Later work extends the PIF terminology to include unexpected Physical InterFerence and intended contACT (PIFACT), which includes interactions that are "active or passive, expected or unexpected" [14]. Their work is implemented on the 52-DoF humanoid WENDY. The ability to maintain gripper orientation when position has been disturbed is shown, and alternately to maintain gripper position by utilizing redundancies in the arm [11]. Compliant robot motions in response to human touch are demonstrated with human-guided movement [46]. Initial work with PIFACT situations classifies different interaction states based on human-human and human-robot contact information [14].

It is worthwhile to note that not all safety measures depend on contact detection and an appropriate behavior response; some approaches instead rely on safe and compliant robot hardware. For example, Lim and Tanie [47] propose end-effector control that is able to tolerate collisions by simply modeling the compliance in a passive viscoelastic robot trunk. In this case however no explicit tactile sensing is performed, and so we refrain from further discussion of such approaches.

\subsection{Interactions that contribute to behavior execution}

We next discuss physical interactions that are a necessary element to the execution of a robot behavior. Here the human-robot contact is deliberate and expected, and does not interfere with the task execution. In many cases the purpose of the behavior is the human-robot contact itself. Approaches treat the interaction as an explicit form of human communication, in most cases either to guide robot behavior or to convey information about the human. In the first case, tactile feedback links to the state of the robot, for example encoding within a finger tap sequence high-level commands for robot control. In the second case, tactile feedback relates to the state of the human, for example conveying human emotion through touch. The goal of detecting human-robot contact in this section is to facilitate communication between a human and robot, for use when executing a robot behavior. 

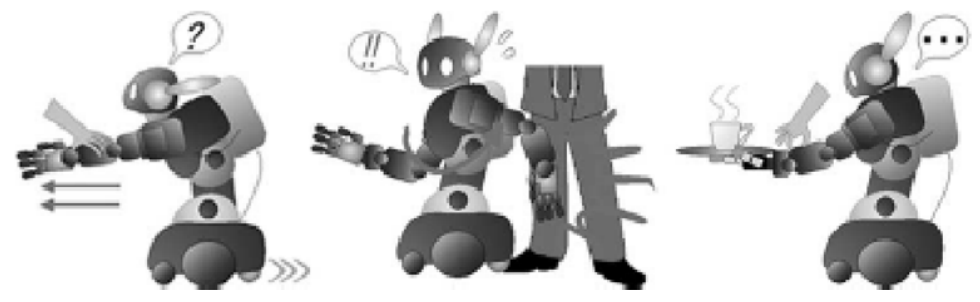

Fig. 11. PIFACT contact in a motivating example [14].

Table 1

Examples of multi-finger contact mappings in Schmid et al. [43].

\begin{tabular}{llll}
\hline Symbol & Hook grasp & Symbol for “Object" & Stop \\
\hline Icon & & & Letter $a$ \\
\hline
\end{tabular}

\subsubsection{Relating to the state of the robot}

Human contact that relates to the state of the robot often is meaningful only within the context of the robot application. For example, tap sequences used for robot behavior selection would convey no meaning to another human.

The work of Voyles and Kholsa [33] infers human user intentions from tactile gestures that are nudges to the robot's end-effector. Nudges are classified, through a preprogrammed mapping, into defined trajectory families, and classification influences the selection of which trajectory to follow. The stated target application for this work is behavior selection within industrial environments, where the robot operator is not a robotics expert and so behavior changes should not require reprogramming the robot. Symbolic force contacts, amounting to a tap alphabet, are inferred for behavior selection in the work of Wosch and Feiten [42]. A broader tactile alphabet is developed in Schmid et al. [43], where multi-finger contact strokes are used for both direct teleoperation as well as higher level robot control via the selection of behavior commands (e.g. Table 1 ).

Other approaches guide motion generation by implementing force-following algorithms that respond to tactile cues produced by a human. The control strategy developed in Schmidt et al. [41] attempts to maximize the number of contact points between the robot gripper and an object (here a human hand), with the result that the gripper and robot arm will follow the contact object as the object moves (Fig. 12). Arm movements generated through human-guided force-following constitutes the demonstration of compliant motion mentioned for the WENDY robot [46]. Note that for both of these works compliant robot behavior is produced, but that the resultant behavior is not remembered by the robot. ${ }^{7}$

\subsubsection{Relating to the state of the human}

In contrast to human contact that relates to the states of the robot, contact that relates to the state of the human typically takes a form that humans would use in the absence of a robotic application, when interacting with another human.

The Communication Robot project of Naya et al. [39] measures human emotion through physical contact between humans and robots, with the goal of developing a robot able to interact with humans through touch. Their work classifies 5 human touching behaviors (slap, stroke, pat, scratch and tickle) from 11 subjects.

\footnotetext{
7 Using the generated motion trajectories as demonstrations within a learning paradigm is cited by Schmidt et al. [41] as a motivation for their force-following work, but no demonstration-based learning is actually implemented.
}

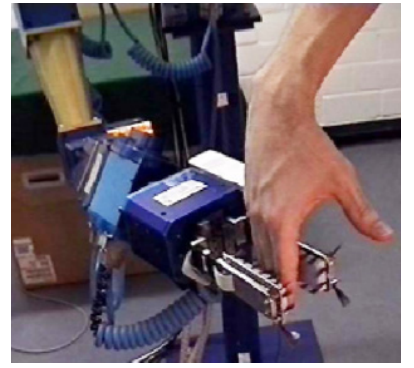

Fig. 12. Motion generation via force following and human guidance in Schmidt et al. [41].

Similarly, Koo et al. [13] aim to identify and classify different types of human touch. Touch classes are automatically determined from the actions of 12 human subjects, guided by the definitions of different contact verbs (e.g. hit, beat, push) and based on contact time, repetition, force and contact area. Note also that the novel tactile sensor of Inaba et al. [40] is able to detect, but not yet classify, human touch.

The series of Robovie humanoid robots, developed with the goal of being partners with humans, utilize tactile feedback in a variety of ways. During behavior execution, tactile feedback triggers interruption by, or the co-execution of, reactive behaviors that respond to touch; for example, the robot turns towards the direction of the touch. Interactive behaviors also utilize touch, for example the robot initiates and sustains a hug only if the human's behavior is classified as being ready to hug and hugging back, respectively [17]. Tactile communication is the primary focus of Robovie-IIS (Fig. 13, left). The position and pose of a human is estimated based exclusively on tactile feedback and is utilized in reactive behaviors, for example using the pose estimation in order to gaze into the face of the human. The mapping from touch to pose is learned from motion capture data, as the robot performs one of over 100 previously developed communication behaviors [18]. A method for the classification of tactile interactions, that furthermore automates the class definitions, is validated by having Robovie-IIS interact with 284 human subjects in a shopping mall [48]. A method for addressing the undesirable issue of highly sensitive tactile sensors detecting the robot's own motion is validated on Robovie-IIF while performing communication behaviors [19]. With Robovie-IV (Fig. 13, right) multi-modal communication - specifically tactile, vision and voice - between a humanoid and human is the focus. The authors categorize interactions into human-robot interaction modes, and a long term study places Robovie-IV into an office environment where the robot seeks out humans for interaction [15].

The interpretation of human intentions becomes all the more relevant when the primary purpose of the robot system is human-robot communication through physical contact. The robotic seal Paro of Shibata and colleagues is a robot in stuffedanimal form with intended therapy applications, specifically for use with elderly patients in a nursing home (Fig. 14, left). ${ }^{8}$ The

8 The Paro images were taken from http://unit.aist.go.jp. 


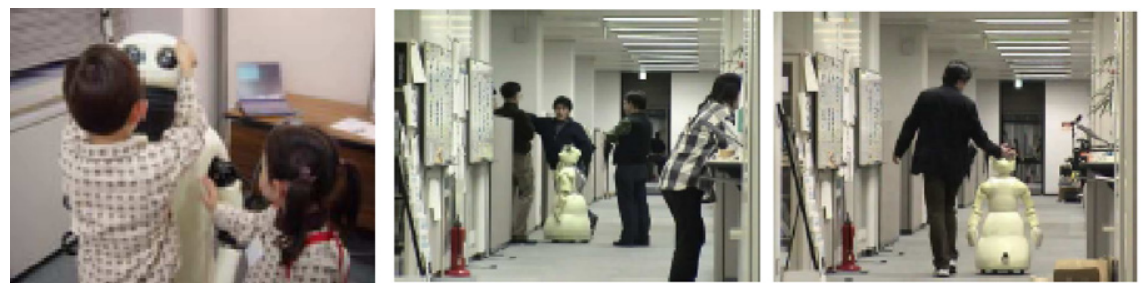

Fig. 13. Tactile interactions between Robovie-IIS and children (left). Mulit-model communication between Robovie-IV and humans within an office environment (right).
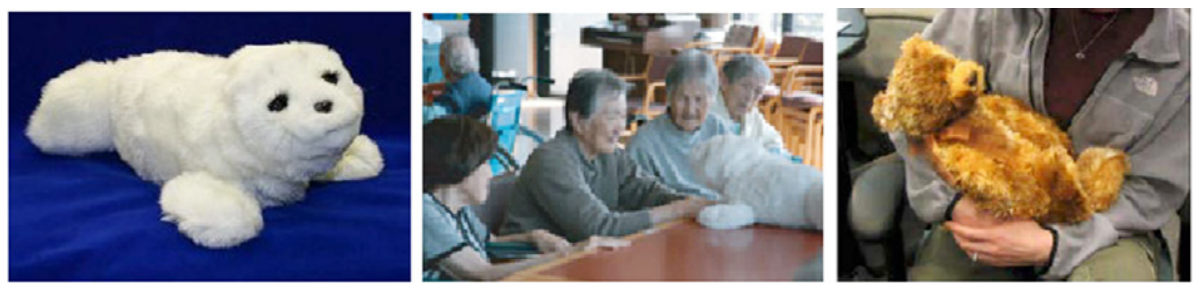

Fig. 14. The Paro (left, center) and Huggable (right) robots as pet surrogates in animal-assisted therapy.

tactile data contributes to the determination of the robot's internal state, which drives the selection and execution of a finite number of hand-coded behaviors, similar to those of a real seal [37]. Of particular note is that the response magnitudes of these behaviors depend on the sensor readings, and furthermore that human touch is classified and used to adaptively update the robot's behavior selection paradigm [28] (see also Section 3.3). An earlier version of the seal had a walking behavior driven by a neural oscillator fed by sensor data (tactile, visual and auditory), and the authors' work with pet robots also includes an early "dog" and "cat" $[37,38]$.

Stiehl et al. [30] also develop a robot as a pet surrogate for animal-assisted therapy. The behaviors of this robot are based entirely on the idea of the robot touching the human back, where through motion in its neck and shoulders the robot teddy bear (the Huggable) is able to orient itself towards the direction of the human touch, and to nuzzle with and hug a human (Fig. 14, right). Initial results classify multiple human touch types, which future work plans to utilize in order to select between robot behaviors. The robotic creature robot recently developed by Yohanan and MacLean [32] senses human touch and communicates back its own internal state via vibrotactile purring, stiffening its ears and breath modulation. Future work with this robot expects to use it as a tool in the study of the role of affective touch, i.e. touch that communicates or evokes emotion, in companionship and anxiety management.

Another body of research interprets tactile interactions with humans in order to match the natural human response to that same interaction. The interactions in this case are between humans and highly anthropomorphic humanoids intended to behave as humans (Fig. 15). A set of androids (Fig. 16) are developed with the goal of generating natural-looking motions on human-like robots, which in particular overcome the uncanny valley [49]. Their soft skin tactile sensor is reported to be human-like in feel and temperature. The android Repliee Q2, developed from the prototype Repliee $\mathrm{R} 1$, is able to distinguish between different types of touch, ranging from stroking to hitting, and is teleoperated through a sophisticated motion capture system to produce natural human-like motion [50]. The geminoid HI-1 aims to serve as a duplicate of a specific human, and sophisticated teleoperation contributes, along with the tactile feedback, to natural interactions with other humans [23].

\subsubsection{Other approaches}

To conclude this section, we discuss approaches that do not strictly address exclusively either the state of the robot or that of the human.
The first set of works involve what is essentially a manipulation task, where a human is the object being manipulated. Here human touch is not active, to guide robot behavior, and neither is it interpreted, to convey any human intentions. Ohmura and Kuniyoshi [27] present a method for lifting heavy objects that depends on whole-body contact between a humanoid robot and heavy object. The contact state between the humanoid and object depends on both the shape and dynamics of the humanoid and the interaction object. By modeling this interaction, the authors develop a method for lifting heavy objects that depends on touch detection, rather than on more powerful motors. The humanoid is reported as able to lift a $30 \mathrm{~kg}$ box, the heaviest in the literature as of publication, as well as a $66 \mathrm{~kg}$ human-shaped dummy (Fig. 17, left). The target application for this work is assistance robots able to lift people, for use in rescue efforts, nursing homes and hospitals. Patches of the skin also are placed on a human and used, in combination with a motion capture system, to gather data for the purpose of developing mechanisms for humanoid control [51]. The ability to lift humans for assistance applications is also the target for the RI-MAN humanoid of Mukai et al. [24], which is able to lift a human dummy of $16 \mathrm{~kg}$ (Fig. 17, right) but has sensing specifications that aim to lift a human of $60 \mathrm{~kg}$.

The second application represents an intersection between tactile feedback that relates to the state of the robot versus the state of the human. The Ms DanceR [35], and later the PBDR [31], perform partner tasks with a human; in particular, partner ballroom dancing tasks (Fig. 18). The human partner is the dancing lead and thus guides the motion of the robot. The robot interprets tactile contact with the human to infer the human's intentions. Specifically, contact with a human dance partner generates a detected force along the $x, y$-axis and moment about $\theta$, which is used to predict the next dance step of the human. Later work improves step prediction by considering also the time series of the force/moment data, as well as encoding a model for human motion based on force and laser-range data [52]. Human stride length is detected according to contact force signatures at the beginning and end of each dance step, and robot stride is scaled to match that length [53]. In addition to entertainment value, humanassistive apparatuses are also a target application of this humanstate prediction technology, for example an assistive walker that predicts and attempts to prevent a human from falling [54].

\subsection{Interactions that develop robot behavior}

We now present physical interactions between a human and robot that serve as a tool to build or adapt robot behaviors. Here 

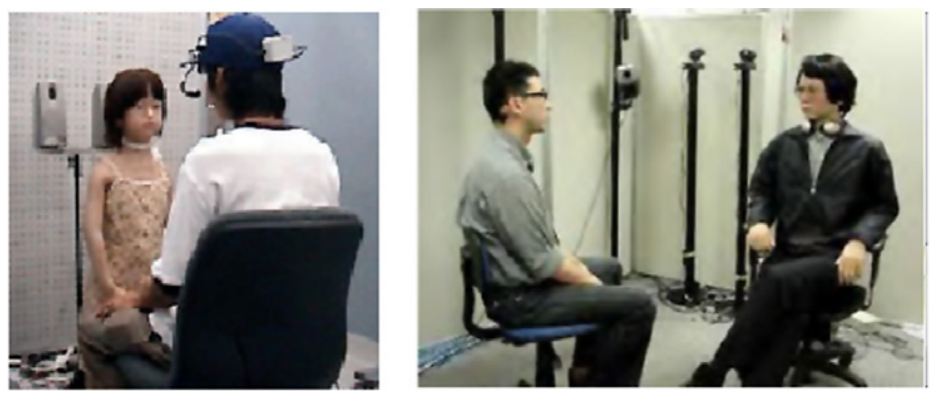

Fig. 15. Human subjects in conversation with the android Repliee R1 (left) and the geminoid H1 (right).
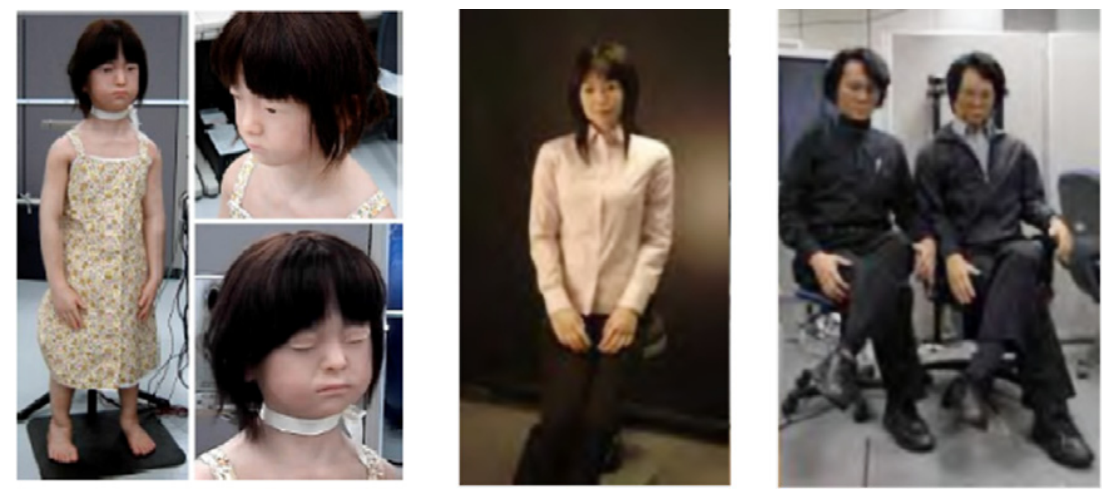

Fig. 16. The android Repliee R1 (left), the android Repliee Q2 (center) and the geminoid H1 (right).
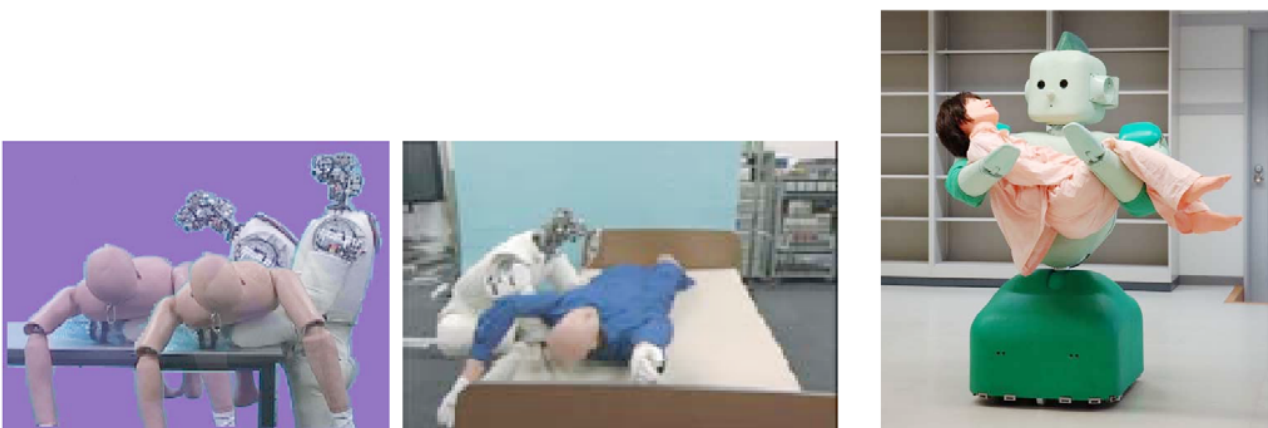

Fig. 17. Humanoid robot lifting a human dummy using whole-body touch detection (left, center, [27]). Humanoid RI-MAN lifting a human dummy (right, [24]).
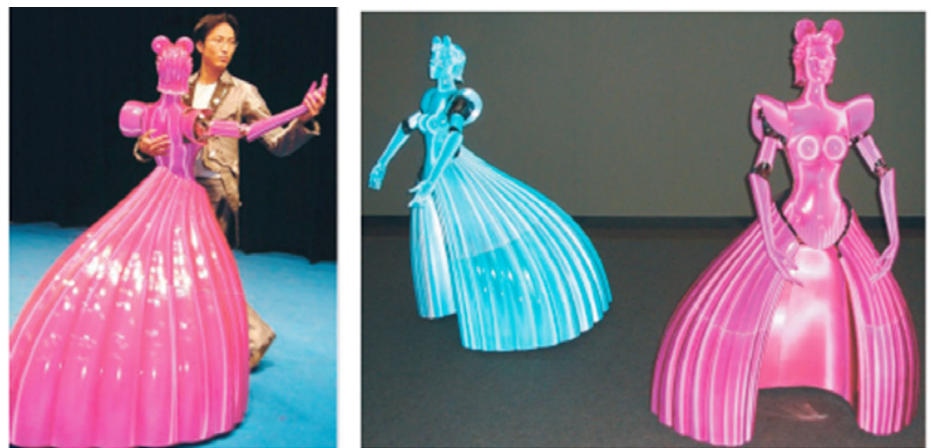

Fig. 18. The ballroom dancing robot PBDR [31].

the tactile sensors are used for instruction or as a learning aid. The human-robot contact is intentional and expected by the robot, and the robot modifies its own behavior in response to the interactions with the human. Human touch in this context also is intended as a form of communication with the robot, which accordingly might require interpretation of the human's intention. In contrast to the works in Section 3.2 that interpret human intention for motion generation, here the resulting behavior is learned. Moreover, the learned behavior will not necessarily expect or rely on human touch at the time of execution, after the learning is complete; rather, the detection and interpretation of touch most often is for behavior development purposes only. The goal of contact with 

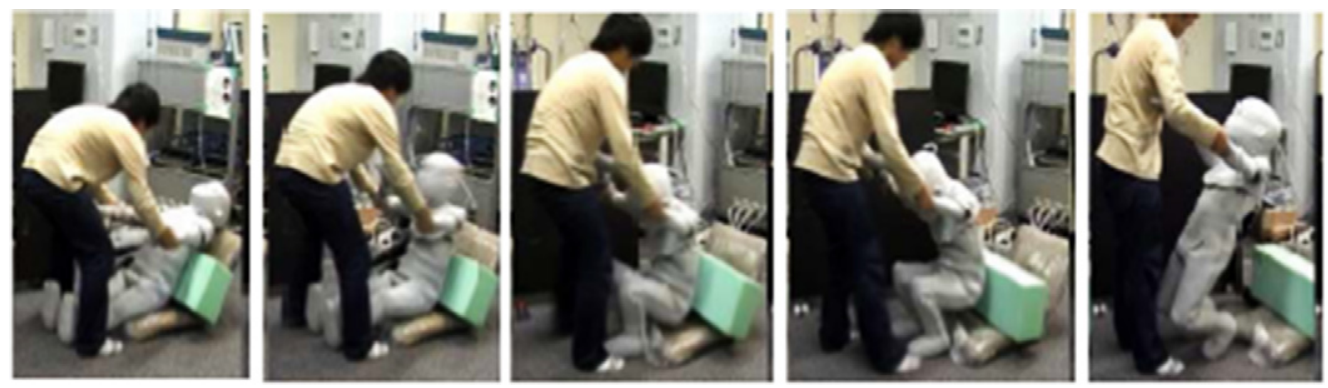

Fig. 19. Behavior learning via "motor development with physical help" with the $C B^{2}$ robot [20].

a human in this section is to produce adaptive and compliant robot behaviors, using a more flexible alternative to behavior development by hand.

Tactile feedback is employed for the development of robot motion behaviors in the "Teaching by Touching" paradigm of DallaLibera et al. [55]. ${ }^{9}$ Here the robot responds to the teacher's touch pattern, and if the response is not what was intended, a means other than touch (e.g. direct manipulation) is used to provide a target pose. The mapping from touch to a change in robot pose is learned, making the technique adaptable to different teachers. In a similar vein, by translating finger swipes on opposing touchpads into small rotational or translational movements, touch is used to iteratively change the pose of the $i C u b$ during the execution of manipulation behaviors in the work of Argall et al. [44]. Here pose corrections are used either to improve a motion behavior learned from human demonstration, or to adapt it to accomplish and learn a different motion task. Earlier work by Grunwald et al. [34] enables a human to easily position and move a large industrial robot arm for demonstration of a piston insertion task, by using tactile feedback to minimize resistance to movement.

The work of Minato et al. [20] employs tactile feedback in order to learn a new robot behavior. The child-sized android $C B^{2}$ explicitly interprets touch feedback from a human, for the purposes of safe operation and behavior learning. The authors introduce the technique of "motor development with physical help", where a human teacher provides physical support and motion guidance to the robot, and the robot attempts to minimize the support forces provided by the human teacher while concurrently learning the resulting motion (Fig. 19). In this work the $C B^{2}$ is taught how to achieve a standing position from a prostrate position, with human assistance. The approach is extended in Ikemoto et al. [56] by having the teacher additionally provide a critique on the timing between posture transitions. Force guidance between key postures also is used for behavior learning with the macra robot in Yoshikai et al. [25]. Here tactile feedback furthermore enables behavior adaptation, where physical human assistance allows the robot to handle obstacles and the resulting motion is learned (Fig. 20).

Other approaches that adapt existing robot behaviors in response to human touch use the tactile sensor readings as a reward signal within a Reinforcement Learning $(R L)$ framework. ${ }^{10}$

\footnotetext{
9 This work differ from all others presented in this article by not having the tactile sensor located on the physical robot body; instead, the robot's behavior is recorded via motion capture, replayed in simulation and the teacher provides corrections via touch screen. However, the work of Minato et al. [45] intends to employ the paradigm to provide touch corrections on the actual BabyBot robot.

10 For a full description of Reinforcement Learning we refer the reader to Sutton and Barto [57]. Note also that Naya et al. [39] put forth a behavior learning idea as future work, which to our knowledge was never completed.
}

RL drives adaptive behavior selection on the Paro robot, where the classification of human touch is used directly for behavior selection. Touch classification additionally is translated into a reward signal, where for example stroking provides a positive reward and beating provides a negative reward. The reward then is used to update the behavior selection parameters, using RL techniques $[28,37]$.

\subsection{Summary}

From the HRI viewpoint, we have identified three primary tactile HRI topic areas: human contact that interferes with robot behavior execution (interference contact), human contact that contributes to behavior execution (contribution contact), and human contact that enables behavior development or adaptation (developmental contact). A summary of the different ways in which physical human-robot contact is used within the current literature is provided in Tables 2 and 3 (How it is used).

Within the topic of interference contact, the state-of-the-art not only detects collisions with a human, but also goes beyond a reactive response to planning a recovery; in particular, a recovery that reasons about the effects of both the collision and task un-fulfillment [14]. Within the topic of contribution contact, the state-of-the-art takes two branches. The first branch uses human touch to directly control robot motion, through teleoperation and behavior selection via a defined tactile alphabet [43] or by guiding compliant robot motion through the use of forcefollowing algorithms $[41,46]$. The second branch infers human intentions - moreover, the intentions of humans within general society (i.e. not robotics researchers) - and uses these inferred intentions to guide and adapt robot behavior [15,28,30]. Advanced work within this branch also goes beyond interpreting the intentions of the human with whom the robot is interacting, to inferring a natural human-like response to the touch [23]. Within the topic of developmental contact, the state-of-the-art uses learning algorithms to exploit human touch for the adaptation or refinement of existing behaviors, as well as for the development of new task-accomplishing behaviors [25,28,44,55,56].

\section{Discussion}

This section concludes our review of the tactile HRI literature to date. We begin by providing a summary of current trends within tactile HRI, identifying approaches which we consider to define the intersection of advanced tactile detection and HRI work within the field. We then highlight open areas for future research, and conclude by delineating those topics not covered in this review. 

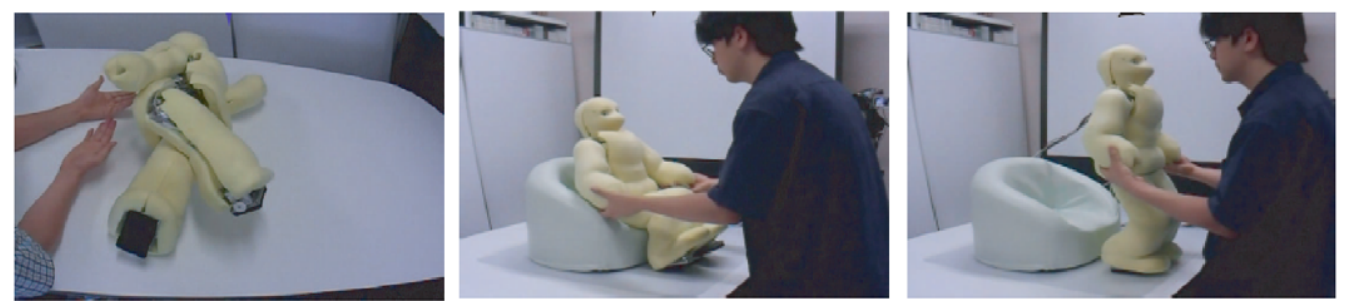

Fig. 20. Behavior learning and adaptation with the macra robot [25].

Table 2

Tactile sensing for Human-Robot Interactions within the literature to date (Part 1: Respond to disturbance, State classification and Behavior selection).

\begin{tabular}{|c|c|c|c|c|c|}
\hline & & & How it is used & & \\
\hline & & & Response to disturbance & State classification & Behavior selection \\
\hline & & & WENDY2000 & Robovie-IIS & Voyles (1995) \\
\hline & & & WENDY2002 & Robovie-IV & MsDanceR \\
\hline & & Ioration & Wosch (2002) & MsDanceR & Robovie-IIF \\
\hline & & Location & Grunwald (2003) & Paro & Robovie-IIS \\
\hline & & & Frigola (2006) & (Haptic Creature) & Robovie-IV \\
\hline & Contact & & QRIO & & \\
\hline & & Area & & $\begin{array}{l}\text { WENDY2005 } \\
\text { Koo (2008) }\end{array}$ & \\
\hline & & & & WENDY2005 & \\
\hline & & & WENDY2000 & Robovie-IIS & Robovie-IIS \\
\hline & & Duration & WENDY2002 & Robovie-IV & \\
\hline & & & & Коo (2008) & \\
\hline What is detected & & & WENDY2000 & WENDY2005 & \\
\hline & & & WENDY2002 & Robovie-IIS & Robovie-IIF \\
\hline & & Magnitude & Wosch (2002) & Robovie-IV & Robovie-IIS \\
\hline & & & Grunwald (2003) & Paro & Robovie-IV \\
\hline & Force & & Frigola (2006) & Koo (2008) & \\
\hline & & Orientation & WENDY2000 & & Voyles (1995) \\
\hline & & Orientation & WENDY2002 & & \\
\hline & & Moment & Grunwald (2003) & MsDanceR & Voyles (1995) \\
\hline & & IVoment & & & MsDanceR \\
\hline & & Static & & & Schmid (2007) \\
\hline & Relational & Dynamic & & & Schmid (2007) \\
\hline & & Vibration & & & \\
\hline & & Temperature & & Huggable & \\
\hline & Other & Kinesthetic & & Huggable & \\
\hline & & Electric field & & Huggable & \\
\hline
\end{tabular}

\subsection{Overview and categorization}

Within Tables 2 and 3 a summary is provided of what is detected during tactile contact with a human, and how that detected information is used by the robot. Note that these categories are intended to summarize what has been detected and used within the literature to date, and do not prescribe the limits of what might be detected or used within future work.

In detail, we subdivide the topic of what is detected, or the elements of tactile contact detected by the robot, into the following categories: contact location, area and duration ${ }^{11}$; force magnitude, orientation and moment; static and dynamic relationships between sensor readings; temperature, vibration, kinesthetics and electric field. Note that readings from a single sensor may map to multiple categories, for example a dielectric material that detects force magnitude and position. Similarly, multiple sensors may map

\footnotetext{
11 Note that to determine contact location for individually placed sensors is immediate (i.e. the location of the sensor), and to determine contact area is typically only possible with skins or arrays of individually placed sensors. For soft skins and sensor arrays, the sensor placement resolution is typically high enough so that determining contact area becomes fairly straightforward. For hard skins the calculation is less immediate but possible, and contact area smaller than the size of a given hard shell panel often is inferred.
}

to a single category, for example the detection of force magnitude by deformation foam and piezo-based sensors.

We also subdivide the topic of how it is used, or in what ways the robot utilizes the detected sensory information. In the category Respond to disturbance, the robot responds to contact that interferes with behavior execution, for example reducing joint force in order to not pinch a trapped object. In State classification the robot uses the detected information to classify internal (i.e. its own) or external (e.g. the human's) state. ${ }^{12}$ In Behavior selection the robot interprets tactile feedback in order to select between existing robot behaviors. ${ }^{13}$ In Behavior execution human contact is an expected part of robot operation, for example the robot being stroked by a patient as a part of animal-surrogate therapy. In Behavior guidance the robot interprets the detected contact as a cue to guide behavior execution, for example the physical guidance of tooltip position. ${ }^{14}$ In Behavior development, the robot responds

12 The use of contact information for State classification often serves as a precursor for its use within Behavior Guidance or Behavior Execution.

13 Behavior Selection works to date select from a discrete set of high-level behaviors, but future work could select from an infinite set of lower-level behaviors or actions.

14 Behavior Guidance works to date all relate to motion, for example forcefollowing or direct robot motion control by a human, but this would not need to be the case with future work. 
Table 3

Tactile sensing for Human-Robot Interactions within the literature to date (Part 2: Behavior execution, Behavior guidance and Behavior development).

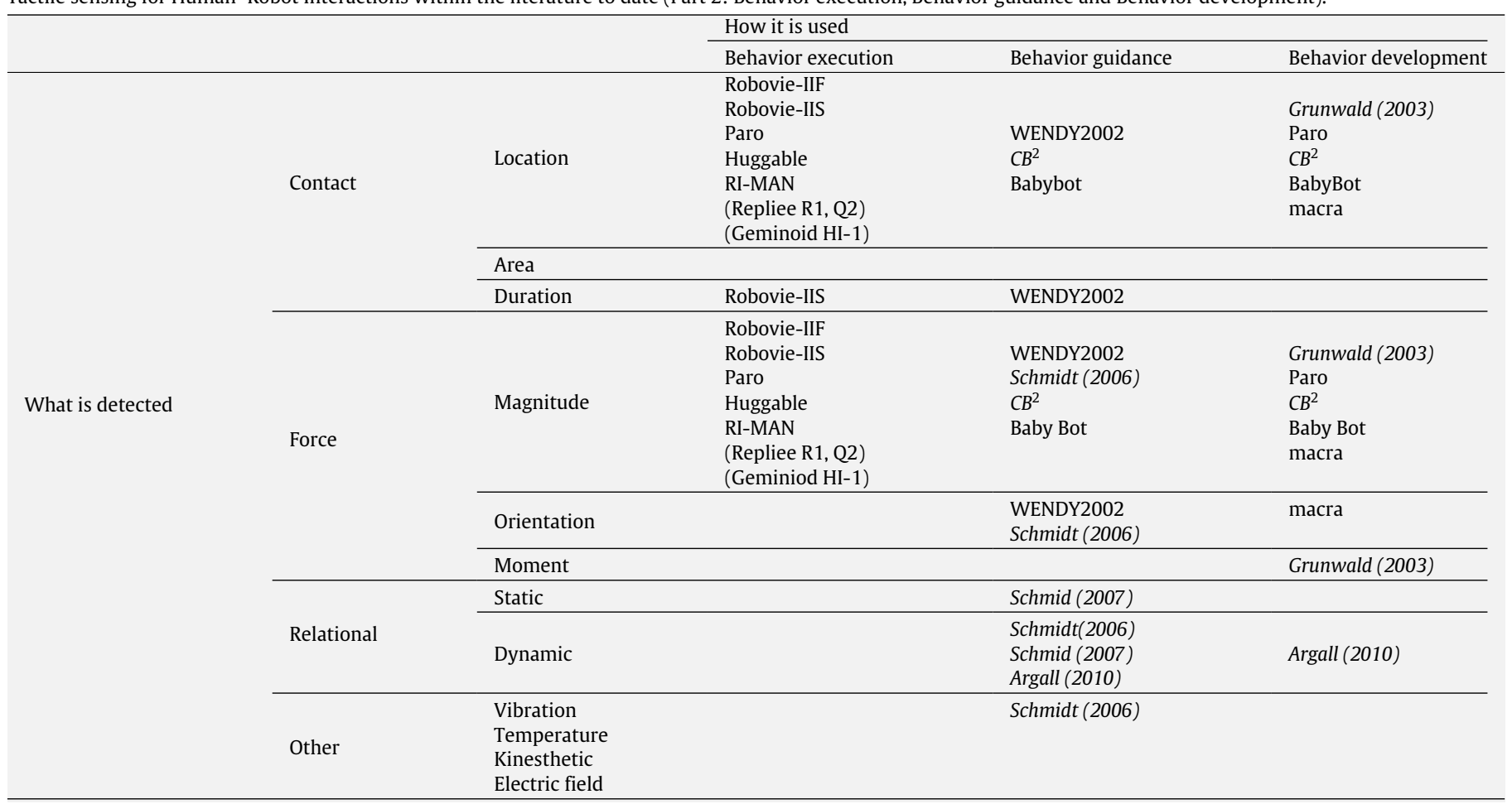

to human contact, for example by changing its behavior selection paradigm or adjusting its pose to minimize contact forces, and then adopts the resultant change in order to adapt an existing behavior or build a new behavior.

\subsection{The intersection of tactile sensing and HRI}

Overlap between the fields of tactile sensing and physical interactions between humans and robots is discussed within this section. We first identify the intersection of the state-of-the-art in both fields, and following this highlight which tactile sensors are appropriate for use within our identified HRI categories.

\subsubsection{Current state-of-the-art}

The applications presented in this paper are placed within our contributed categorization in Table 4. Again, approaches are grouped by HRI contact type (Interference, Contribution, Developmental), as well as by tactile detection technique (Hard skins, Soft skins, Not skin-based). For state-of-the-art works from the tactile and HRI viewpoints, note the 'a' and 'b' markers respectively. Here we highlight the intersection of advanced work from both the HRI and tactile-sensing viewpoints.

The humanoid Robovie-IV has a soft force-sensing skin covering the entire robot body, operates within a real-world office environment, and uses tactile feedback to both select and perform a multitude of active and reactive interaction behaviors with the goal of communication with, as well as safe operation around, humans [15]. Two separate projects have developed robotic pet surrogates for animal-assisted therapy that focuses on tactile interactions. The seal-shaped robot Paro has a soft force-sensing skin plus fur covering its entire body, and uses tactile feedback for behavior selection, to adjust behavior execution and to adapt its behavior selection paradigm according to a learning algorithm [28]. The Huggable has a soft multi-modal (force, electric field, temperature, kinesthetics) sensory skin plus fur covering its entire teddy-bear-shaped body, and classifies human touch to both select and perform tactile interactive behaviors [30].
Table 4

Tactile HRI literature, placed within our categorization.

\begin{tabular}{|c|c|c|c|}
\hline & Hard skins & Soft skins & Not skin-based \\
\hline Interference & $\begin{array}{l}\text { WENDY2000 } \\
\text { Frigola }(2006)\end{array}$ & WENDY2005 ${ }^{\mathrm{b}}$ & $\begin{array}{l}\text { Wosch (2002) } \\
\text { Grunwald (2003) } \\
\text { QRIO }\end{array}$ \\
\hline Contribution & $\begin{array}{l}\text { WENDY2002 } \\
\text { Koo (2008) }\end{array}$ & $\begin{array}{l}\text { Robovie-IIF }^{\mathrm{a}} \\
\text { Robovie-IIS } \\
\text { Robovie-IV }^{\mathrm{ab}} \\
\text { Repliee R1 } \\
\text { Repliee Q2 } \\
\text { Geminoid HI-1 }^{\mathrm{b}} \\
\text { Huggable }^{\mathrm{a} b} \\
\text { Paro }^{\mathrm{ab}} \\
\text { Ohmura }^{\mathrm{a} 2006)^{\mathrm{a}}} \\
\text { RI-MAN }^{\mathrm{a}}\end{array}$ & $\begin{array}{l}\text { Voyles (1995) } \\
\text { Inaba (1996) } \\
\text { Naya }(1999) \\
\text { Wosch }(2002) \\
\text { Ms DanceR } \\
\text { Robovie-II } \\
\text { Schmidt }(2006)^{\mathrm{b}} \\
\text { Schmid }(2007)^{\mathrm{b}} \\
\text { PBDR }^{\mathrm{b}} \\
\text { Haptic Creature }^{\text {Hapt }}\end{array}$ \\
\hline Developmental & & $\begin{array}{l}\text { Paro }^{a b} \\
\mathrm{CB}^{\mathrm{ab}} \\
\text { macra }^{\mathrm{ab}}\end{array}$ & $\begin{array}{l}\text { Grunwald (2003) } \\
\text { Argall }(2010)^{\mathrm{b}} \\
\text { BabyBot }^{\mathrm{a}}\end{array}$ \\
\hline
\end{tabular}

a Tactile state-of-the-art.

The child-sized android $C B^{2}$ is covered entirely in soft forcesensing skin, classifies human touch to perform active and passive behaviors and furthermore learns physical behaviors from force interactions with a human teacher [20] and adapts the learned behavior based on teacher feedback [56]. The smaller humanoid macra similarly is covered entirely in a soft force-sensing skin and learns behavior from force-guidance by a human teacher, and furthermore adapts existing behaviors by responding to human touch provided for assistive purposes [25]. ${ }^{15}$

\footnotetext{
15 Note that the future work proposed in Minato et al. [45] to implement the "Teaching by Touching" paradigm of DallaLibera et al. [55] on the BabyBot also would represent an intersection between the state-of-the-art in both fields.
} 


\subsubsection{Connecting sensors and interactions}

Here we provide a discussion of the types of sensors that are appropriate for use within each of our identified HRI categories; that is, for detecting human contact that interferes with (Interference) or contributes to (Contribution) robot behavior execution or development (Developmental).

Applications that aim to detect Interference interactions, that disrupt robot task execution, at a minimum must detect contact, which all tactile sensors reported within this survey do. Extensive coverage furthermore is important, such that all parts of the robot that might physically touch the human are capable of detecting the contact.

- Reactive motions in general require knowledge of the contact location, and often also orientation, which is possible to infer under a variety of force sensor setups (e.g. [11,25,33]).

- For sophisticated responses to the contact, multi-modal sensing or detection at a high spatial resolution might be required. Multi-modal sensing is typically achieved through either soft skins or uncovered sensors placed on the robot's body, but not with hard skins. High spatial resolution can be accomplished within any of the sensor categories: hard or soft skins, and setups that are not skin-based.

- Softness in feel might be important to mitigate the possibility of injuring the human, or for shock absorption to protect the robot. Softness can be achieved with deformable coverings (e.g. urethane foam [15]).

- Lastly, we note that the contact might be anticipated, and subsequently avoided, if some sort of extended contact sensor (e.g. on whiskers, as in [37]) or proximity sensor (which is beyond the scope of this review; see Section 4.4) is employed.

Applications that target the detection of Contribution interactions, that are a key element in the execution of the robot behavior, typically require more information than simply contact detection. For interactions that relate to the state of the robot, the human generally tailors their touch to have particular meaning to the robot.

- Force-following requires knowledge of the force orientation, and possibly also magnitude, in addition to contact location. Again, such information might be gathered under a variety of force sensor setups (e.g. [11,25,33]). Similar specifications typically are required for nudge detection.

- Symbolic touches for behavior selection possibly require knowledge of the contact duration (e.g. using the PVDF-based silicone skin of Miyashita et al. [18]) or relational information (e.g. using the resistive pressure sensor array of Schmid et al. [43]).

For interactions that relate to the state of the human, the intent of the human's touch often has a broader meaning than simply conveying information to a robot, for example touches that are a natural expression of the emotional state of the human.

- Relational, vibration and/or kinesthetic information can help distinguish between different types of touch (e.g. stroking vs. slapping), which requires a high spatial resolution and sampling rate for the sensor. These two goals might conflict with each other if the high spatial resolution is achieved with a large number of sensors, which accordingly increases the time of a single sampling sweep.

- Contact location may or may not be important, depending on if the location has any significance for what the human is communicating.

- It might be necessary to distinguish the human as the source of the contact (e.g. by detecting temperature or electric field [30]).

- It is possible that the human will communicate with a light touch, that requires very sensitive detection capabilities, which frequently has the trade-off of being highly subject to noise or detecting the self-motion of the robot.
Applications that aim to detect Developmental interactions, that use tactile feedback to build and adapt robot behaviors, also require more sophisticated sensing than simply contact detection.

- If the tactile feedback is interpreted as a reward, different types of touch might be mapped to different reward signals. For example, the strength of the touch might correlate to size of reward, in which case detecting force magnitude or contact duration would be necessary.

- If the tactile feedback is used for motion guidance via forcefollowing in order to learn motion behaviors, the requirements will be the same as those noted previously (in the discussion of Contribution interactions).

We conclude with a note about highly sensitive tactile sensors, in particular within the context of soft skins. To detect human touch can require high sensitivity, especially when detecting touches that exhibit minimal force or contact area. With highly responsive sensors, however, the issue of self-induced activations becomes more prevalent: the more sensitive the sensor, the more likely the robot is to detect its own movement. Thus, as sensors become increasingly sensitive, and cover larger portions of the robot body, distinguishing between self-induced activations and other activations, including human touch, becomes a necessity. Some works do take steps to address this issue, for example the study of noise modeling and subtraction in Tajika et al. [19] and the approach, proposed in Minato et al. [20], of combining a temporal behavior component with self-movement knowledge.

\subsection{Areas open for future research}

This section identifies some open areas for future research within the field of tactile HRI. We expect that each of the HRI categories defined within this article will continue to be important areas for future research, and thus structure the following discussion according to these categories.

\subsubsection{Unexpected or unintended interactions}

As the presence of robots within general society increases, we anticipate that the consideration of unexpected interactions between humans and robots, that moreover interfere with robot behavior execution, will gain increasing importance. An appropriate response by the robot to unexpected physical interactions will be necessary for safe robot operation around humans, as well as for successful robot operation in general.

Beyond improvements in collision detection and reactive behaviors, one direction for future work is to use the contact information in a more sophisticated manner, thus expanding upon ideas presented by Iwata and colleagues $[11,14]$. For example, if the effects of detected human-robot contacts are known and represented within a planning framework, then producing a plan that both recovers from a collision and completes an interrupted task becomes feasible. A contact model within a planning framework also might serve to halt a sequential chain of undesirable contacts. For example, if coming into contact with an outstretched human arm is frequently followed by full-body collision with the human, then the robot's response to the first contact could reason about avoiding the full-body contact expected to follow.

Another avenue open for future research with interference contact is to consider persistent physical interactions, that perhaps interfere with task completion permanently rather than just temporarily. By contrast, works to date have focused on temporary interferences that are sudden, unexpected and of brief duration.

Lastly, interference research would benefit from the use of more sophisticated sensing techniques. The work of Iwata and colleagues represents the state-of-the-art from the viewpoint of 
interference interactions, but not from the viewpoint of tactile sensing. While their work covers the arms and shoulders of a humanoid with a soft skin of force sensors, the state-of-theart would cover the entire robot body in the sensor skin and furthermore employ multiple sensor modalities.

\subsubsection{Interpretation of human intentions}

When considering robot operation around, and interaction with, those who are not robotics experts, the interpretation of natural human intentions becomes particularly relevant. The intersection of advanced techniques from both the HRI and tactile sensing viewpoints is well-represented in this category, with the pet surrogate robots Paro and Huggable. The target application domain for both of these robots is animal-assisted therapy.

We suggest the interpretation of natural human intentions for non-therapy applications as a relevant area for future investigation as well. The deployment of Robovie-IV within an office environment is one such application, and others could include private environments like the home, or public environments such as restaurants and stores. Of particular interest would be for android and geminoid robots to have tactile interactions with humans in situations beyond seated conversations, and moreover outside of laboratory or exposition settings.

Regarding the deployment of robots within novel human environments, especially those that are public, sensors with high spatial resolution and complete coverage of the robot body will be particularly valuable for the interpretation of human intentions. Sensors that aim to mimic the sensing capabilities of the human skin are especially appropriate for robots that aim to mimic the behavior of humans. To mimic natural human behavior, we expect that detection of the tactile interactions that humans have with other humans will be a necessary, if not sufficient, criterion. The idea here is that similar sensing capabilities would enable similar touch detection, and from this information a similar interpretation of the tactile interactions might be produced.

\subsubsection{Human-guided behavior development}

Given the expectation of robots operating around humans, and therefore human presence around robots, exploiting the knowledge and abilities of a human is a practical approach for producing robot behaviors. Touch is one form of communication through which a human can transfer knowledge to a robot. Comparatively little work to date has addressed how human touch might be employed to produce robot behaviors.

One possible approach is to exploit the human for behavior guidance, for example as seen in the generation of compliant robot motions [46] and human-guided motion trajectories [41]. When used for behavior development however, the robot would learn the behavior produced by human guidance, and the guidance thus would not be required every time the robot operates. Works that step in this direction have guided transitions between robot postures $[20,25,56]$ or provided corrections on a robot pose [44,55], but none have used tactile guidance to generate complete robot motion behaviors for learning.

In addition to using tactile feedback to shape behaviors physically, through guided motion, feedback might also shape behaviors abstractly, through a learning paradigm. Here touch might be interpreted as a feedback signal, for use within a learning algorithm. The are many ways to interpret a tactile signal for use within a learning paradigm, with reward being one example [28]. With multi-modal sensing the options for signal interpretation expand, since more complex sensory feedback enables more complex feedback signals for the learning algorithm. If the sensory signal is classified, multi-modal sensing also provides a larger number of potential tactile classes.
To conclude, we note that most research to date falls within the categories of contact that interferes with, and contact that contributes to, robot behavior execution. We therefore consider topics outside of these areas, such as behavior development, to be particularly deserving of future investigation.

\subsubsection{Directions within tactile sensing}

To summarize the content of the previous sections from a tactile sensing viewpoint, we identify the following topics as particularly useful areas for future tactile sensing research:

- Continuous coverage. Sensor coverage that is continuous, and furthermore spans the entire robot body, is particularly relevant for safe and effective robot operation within real world environments.

- Minimal customization. The development of technologies that attain continuous coverage with minimal custom-fitting for physically different robot platforms is quite practical and furthermore relatively unaddressed.

- Mass production. The mass production, or industrialization, of sensor technologies will contribute to the development of sensors that are more affordable, rugged and reliable, and available in a greater variety.

- Biological inspiration. The explicit imitation of the sensing capabilities in the human skin is of particular relevance for applications that aim to reproduce a human-like response or behavior.

- Multi-modality. The ability to sense from multiple modalities, with increasingly fine spatial resolution, provides more sophisticated sensor data, which in turn allows for the development of more sophisticated robot behaviors.

Regarding multi-modality, a potentially large amount of information is contained within a physical contact, and a variety of sensors may be employed to extract different elements of interest. While simple force sensing can determine contact presence, information like temperature and electric field can determine whether the contact comes from a human, vibration can provide an indication about the contact dynamics, and pressure an indication of contact magnitude and duration, for example.

The idea of multi-modality - of combining multiple contact sensors - relates closely to the topic of reproducing the multiple sensing modalities of the human skin. The range of sensations detected by the human skin includes temperature, touch, pressure, vibration and tissue injury. Beyond combining multiple contact sensors, another open area is to furthermore combine local tactile data with information from sensors that detect remote, rather than local, aspects of the world. Such an approach could prove useful, even necessary, for robot behaviors that go beyond reactive responses to contact; for example, that reason about contact within a planning framework.

\subsection{Scope of this review}

We have limited the scope of this review to applications that detect human touch within human-robot interactions. Topics that were not addressed included the following:

1. Tactile sensors for non-HRI applications. This includes the use of joint force-torque sensors and skins of tactile sensors within industrial applications (e.g. for the avoidance of non-human obstacles).

2. Human-centric tactile sensors located externally to the robot body (e.g. a remote touchscreen interface for human control of the robot).

3. The detection of human presence, but not contact. This includes proximity sensors, even those in skin format (e.g. [58,59]), and the use of vision for presence (not contact) detection. 
4. Robot behaviors that incorporate tactile feedback and interact with humans, but do not come into physical contact with the human.

5. Studies that focus exclusively on the effect of robot touch on the human (e.g. [60]).

6. HRI applications with individual contact sensors only.

The second restriction includes most haptics applications within robotics, where by haptics we refer to tactile interfaces for a human user [61]. In contrast to most haptics applications [62], this review has focused on tactile feedback as used by the robot, within robot behaviors, rather than by the human. Moreover, the work presented in this review was restricted to have a human be the source of a touch detected by the robot; that is, the detected contact is the result of a human touching the robot's body. The few haptics applications included in this review therefore had the tactile interface located directly on the robot body (e.g. [43]).

The fourth restriction refers to behaviors, perhaps performed with a human partner or taught by a human instructor, that furthermore do incorporate tactile feedback, but from a nonhuman source. We adopted this restriction because the interaction does not involve physical contact between the human and robot. For example, the humanoid Robonaut has tactile sensors in its hands and is intended to perform partner manipulation tasks, such as carrying tools to a work site, with a human astronaut [63]. The human cooperation aspect makes the task an HRI behavior, and the sensors in the hand provide a tactile element, but since the robot does not touch the human, the work falls outside of what we consider here to be tactile HRI. Another example is to have a human demonstrate a behavior through non-tactile means, for example teleoperation via remote joystick, to a robot that uses tactile sensors to perform the behavior learned from these demonstrations $[64,65]$. Again, the human-robot interaction is decoupled from the tactile interaction, and so we considered such work to be beyond the scope of this review.

The sixth restriction admittedly does exclude a subset of works that fit within our definition of tactile HRI. In particular, this restriction excludes robot applications that detect contact and operate around humans, and thus potentially come into contact with humans. Some applications assume that the source of a detected contact is human, for example the Sony AIBO robot dog [66] has a commercial learning algorithm that interprets a contact signal as a positive human reaction to the robot behavior. Other applications do not reason at all about the source of the contact; for example, while operating in the home around humans, the Roomba ${ }^{16}$ vacuuming robot treats all contacts as obstacles to be avoided. HRI applications with individual contact sensors do contribute to the foundations of the field of tactile HRI. The stateof-the-art, however, for the most part has moved beyond binary contact detection. ${ }^{17}$ As the volume of research within the area of binary HRI contact is large, to address these works in full would have distracted from our focus on recent advances in tactile HRI. We therefore have included only applications of tactile HRI that employed a sophisticated sensing technique (e.g. more than just a single on/off signal), a sophisticated use of sensory data within the behavior (e.g. not just a binary detection of human presence), or both.

\footnotetext{
16 http://www.irobot.com.

17 With the exception of high-resolution arrays of binary contact points.
}

\section{Conclusion}

We have presented a review of current work within the field of Tactile Human-Robot Interactions (Tactile HRI). The detection of human touch can be important for safe robot operation around humans and furthermore may contribute to robot behavior execution, for example in human-guided motion, as well as to robot behavior development, for example a tactile reward within a learning framework. We have addressed the topic of tactile HRI from the viewpoints of two independent research lines within robotics, and have contributed a framework for the categorization of tactile HRI research within each. The first viewpoint categorizes approaches at a lower level, according to the sensor devices employed for contact detection. The second viewpoint categorizes approaches at a higher level, according to the nature of the human-robot contact and how the detected contact is used by the robot during behavior execution or development. Within each category, the state-of-the-art has been identified. To conclude, we have provided a discussion of which sensors are appropriate for use within our identified HRI categories, and also highlighted open areas for future research.

\section{Acknowledgements}

The research leading to these results has received funding from the European Community's Seventh Framework Programme FP7/2007-2013 - Challenge 2 - Cognitive Systems, Interaction, Robotics under grant agreement no. [231500]-[ROBOSKIN].

\section{References}

[1] H. Nicholls, M. Lee, A survey of robot tactile sensing technology, The International Journal of Robotics Research 8 (3) (1989) 3-30.

[2] M. Lee, Tactile sensing: new directions, new challenges, The International Journal of Robotics Research 19 (7) (2000) 636-643.

[3] R.D. Howe, Tactile sensing and control of robotic manipulation, Journal of Advanced Robotics 8 (3) (2003) 245-261.

[4] J. Tegin, J. Wikander, Tactile sensing in intelligent robotic manipulation, Industrial Robot: an International Journal 32 (1) (2005) 64-70

[5] R.S. Dahiya, G. Metta, M. Valle, G. Sandini, Tactile sensing - from humans to humanoids, Transactions on Robotics 26 (1) (2010).

[6] J. Scholtz, Theory and evaluation of human robot interactions, in: Proceedings of the 36th Annual Hawaii International Conference on System Sciences, 2003.

[7] A.D. Santis, B. Siciliano, A.D. Luca, A. Bicchi, An atlas of physical human-robot interaction, Mechanism and Machine Theory 43 (3) (2008) 253-270.

[8] K. Dautenhahn, I. Werry, A quantitative technique for analysing robot-human interactions, in: Proceedings of the IEEE/RSJ International Conference on Intelligent Robots and Systems, IROS'02, 2002.

[9] A. Steinfeld, T. Fong, D. Kaber, M. Lewis, J. Scholtz, A. Schultz, M. Goodrich, Common metrics for human-robot interaction, in: First Annual Conference on Human-Robot Interactions, HRI'06, 2006.

[10] H. Yanco, J. Drury, Classifying human-robot interaction: an updated taxonomy, in: IEEE International Conference on Systems, Man and Cybernetics, SMC'04, 2004.

[11] H. Iwata, H. Hoshino, T. Morita, S. Sugano, Human-humanoid physical interaction realizing force following and task fulfillment, in: Proceedings of the IEEE/RSJ International Conference on Intelligent Robots and Systems, IROS'00, 2000.

[12] M. Frigola, A. Casals, J. Amat, Human-robot interaction based on a sensitive bumper skin, in: Proceedings of the IEEE/RSJ International Conference on Intelligent Robots and Systems, IROS'06, 2006.

[13] S. Koo, J.G. Lim, D. soo Kwon, Online touch behavior recognition of hard-cover robot using temporal decision tree classifier, in: IEEE International Symposium on Robot and Human Interactive Communication, RO-MAN'08, 2008.

[14] H. Iwata, S. Sugano, Human-robot-contact-state identification based on tactile recognition, IEEE Transactions on Industrial Electronics 52 (6) (2005) 1468-1477.

[15] N. Mitsunaga, T. Miyashita, H. Ishiguro, K. Kogure, N. Hagita, RobovieIV: a communication robot interacting with people daily in an office, in: Proceedings of the IEEE/RSJ International Conference on Intelligent Robots and Systems, IROS'06, 2006. 
[16] H. Ishiguro, T. Ono, M. Imai, T. Maeda, T. Kanda, R. Nakatsu, Robovie: an interactive humanoid robot, Industrial Robot: An International Journal 28 (6) (2001) 498-504.

[17] T. Kanda, H. Ishiguro, M. Imai, T. Ono, Development and evaluation of interactive humanoid robots, in: Human Interactive Robot for Psychological Enrichment, Proceedings of the IEEE 92 (11) (2004) 1848-1855 (special issue).

[18] T. Miyashita, T. Tajika, H. Ishiguro, K. Kogure, N. Hagita, Haptic communication between humans and robots, in: Robotics Research. Vol. 28, Springer-Verlag, Berlin, Heidelberg, Germany, 2007, pp. 525-536.

[19] T. Tajika, T. Miyashita, H. Ishiguro, N. Hagita, Reducing influence of robot's motion on tactile sensor based on partially linear model, in: Proceedings of the IEEE/RSJ International Conference on Intelligent Robots and Systems, IROS'08, 2008.

[20] T. Minato, Y. Yoshikawa, T. Noda, S. Ikemoto, H. Ishiguro, M. Asada, CB2: a child robot with biomimetic body for cognitive developmental robotics, in: Proceedings of the IEEE/RSJ International Conference on Intelligent Robots and Systems, IROS'07, 2007.

[21] T. Minato, M. Shimada, H. Ishiguro, S. Itakura, Development of an android robot for studying human-robot interaction, in: Innovations in Applied Artificial Intelligence, in: Lecture Notes in Computer Science, Springer-Verlag, Berlin, Heidelberg, Germany, 2004, pp. 424-434.

[22] D. Matsui, T. Minato, K.F. MacDorman, H. Ishiguro, Generating natural motion in an android by mapping human motion, in: Proceedings of the IEEE/RSJ International Conference on Intelligent Robots and Systems, IROS'05, 2005.

[23] S. Nishio, H. Ishiguro, N. Hagita, Geminoid: Teleoperated android of an existing person, in: A.C. de Pina Filho (Ed.), Humanoid Robots: New Developments, I-Tech, Vienna, Austria, 2007 (Chapter 20).

[24] T. Mukai, M. Onishi, T. Odashima, S. Hirano, Z. Luo, Development of the tactile sensor system of a human-interactive robot RI-MAN, IEEE Transactions on Robotics 24 (2) (2008) 505-512.

[25] T. Yoshikai, M. Hayashi, Y. Ishizaka, T. Sagisaka, M. Inaba, Behavior integration for whole-body close interactions by a humanoid with soft sensor flesh, in: Proceedings of the IEEE-RAS International Conference on Humanoids Robots, HUMANOIDS'07, 2007.

[26] Y. Ohmura, Y. Kuniyoshi, A. Nagakubo, Conformable and scalable tactile sensor skin for curved surfaces, in: Proceedings of the IEEE International Conference on Robotics and Automation, ICRA'06, 2006.

[27] Y. Ohmura, Y. Kuniyoshi, Humanoid robot which can lift a $30 \mathrm{~kg}$ box by whole body contact and tactile feedback, in: Proceedings of the IEEE/RSJ International Conference on Intelligent Robots and Systems, IROS'07, 2007.

[28] K. Wada, T. Shibata, Social effects of robot therapy in a care house - change of social network of the residents for two months -, in: Proceedings of the IEEE International Conference on Robotics and Automation, ICRA'07, 2007.

[29] T. Shibata, Ubiquitous surface tactile sensor, in: First IEEE Technical Exhibition Based Conference on Robotics and Automation, TExCRA'04, 2004.

[30] W.D. Stiehl, J. Lieberman, C. Breazeal, L. Basel, L. Lalla, M. Wolf, The design of the Huggable: a therapeutic robotic companion for relational, affective touch, in: Proceedings of the AAAI Fall Symposium on Caring machines: AI in Eldercare, 2005.

[31] J.-J. Aucouturier, K. Ikeuchi, H. Hirukawa, S. Nakaoka, T. Shiratori, S. Kudoh, F. Kanehiro, T. Ogata, H. Kozima, H.G. Okuno, M.P. Michalowski, Y. Ogai, T. Ikegami, K. Kosuge, T. Takeda, Y. Hirata, Cheek to chip: dancing robots and Al's future, IEEE Intelligent Systems 23 (2) (2008).

[32] S. Yohanan, K. MacLean, A tool to study affective touch: Goals \& design of the haptic creature, in: Proceedings of ACM SIGCHI Conference. Spotlight on Works in Progress, 2009.

[33] R. Voyles, P. Kholsa, Tactile gestures for human/robot interaction, in: Proceedings of the IEEE/RSJ International Conference on Intelligent Robots and Systems, IROS'95, 1995.

[34] G. Grunwald, G. Schreiber, A. Albu-Chaffer, G. Hirzinger, Programming by touch: the different way of human-robot interaction, IEEE Transactions on Industrial Electronics 50 (4) (2003) 659-666.

[35] K. Kosuge, T. Hayashi, Y. Hirata, R. Tobiyama, Dance partner robot-Ms DanceR, in: Proceedings of the IEEE/RSJ International Conference on Intelligent Robots and Systems, IROS'03, 2003.

[36] Y. Kuroki, T. Fukushima, K. Nagasaka, T. Moridaira, T. T. Doi, J. Yamaguchi, A small biped entertainment robot exploring human-robot interactive applications, in: IEEE International Symposium on Robot and Human Interactive Communication, RO-MAN'03, 2003.

[37] T. Shibata, K. Tanie, Physical and affective interaction between human and mental commit robot, in: Proceedings of the IEEE International Conference on Robotics and Automation, ICRA'01, 2001.

[38] T. Shibata, M. Yoshida, J. Yamato, Artificial emotional creature for humanmachine interaction, in: Proceedings of the IEEE International Conference on Systems, Man and Cybernetics, SMC'97, 1997.

[39] F. Naya, J. Yamato, K. Shinozawa, Recognizing human touching behaviors using a haptic interface for a pet-robot, in: IEEE International Conference on Systems, Man, and Cybernetics, SMC'99, 1999.

[40] M. Inaba, Y. Hoshino, K. Nagasaka, T. Ninomiya, S. Kagami, H. Inoue, A fullbody tactile sensor suit using electrically conductive fabric and strings, in: Proceedings of the IEEE/RSJ International Conference on Intelligent Robots and Systems, IROS'96, 1996.
[41] P.A. Schmidt, E. Maél, R.P. Würtz, A sensor for dynamic tactile information with applications in human-robot interaction and object exploration, Robotics and Autonomous Systems 54 (2006) 1005-1014.

[42] T. Wosch, W. Feiten, Reactive motion control for human-robot tactile interaction, in: Proceedings of the IEEE International Conference on Robotics and Automation, ICRA'02, 2002.

[43] A. Schmid, M. Hoffmann, H. Worn, A tactile language for intuitive human-robot communication, in: Proceedings of the Ninth International Conference on Multimodal Interfaces, ICMI'07, 2007.

[44] B.D. Argall, E.L. Sauser, A.G. Billard, Tactile guidance for policy refinement and reuse, in: 9th IEEE International Conference on Development and Learning, ICDL'10, 2010.

[45] T. Minato, F. DallaLibera, S. Yokokawa, Y. Nakamura, H. Ishiguro, E. Menegatti, A baby robot platform for cognitive developmental robotics, in: Proceedings of the IEEE/RSJ International Conference on Intelligent Robots and Systems, IROS'09, 2009.

[46] H. Iwata, S. Sugano, Whole-body covering tactile interface for human-robot coordination, in: Proceedings of the IEEE International Conference on Robotics and Automation, ICRA'02, 2002.

[47] H. Lim, K. Tanie, Collision-tolerant control of human-friendly robot with viscoelastic trunk, IEEE Transactions on Mechatronics 4 (4) (1999) 417-427.

[48] T. Tajika, T. Miyashita, H. Ishiguro, N. Hagita, Automatic categorization of haptic interactions - what are the typical haptic interactions between a human and a robot? in: Proceedings of the IEEE-RAS International Conference on Humanoids Robots, HUMANOIDS'06, 2006.

[49] M. Mori, Bukimi no tani [the uncanny valley], Energy 7 (4) (1970) 33-35 (in Japanese).

[50] H. Ishiguro, Android science - Toward a new cross-interdisciplinary framework, in: Robotics Research. Vol. 28, Springer-Verlag, Berlin, Heidelberg, Germany, 2007, pp. 118-127.

[51] K. Ogata, D. Shiramatsu, Y. Ohmura, Y. Kuniyoshi, Analyzing the knack of human piggyback motion based on simultaneous measurement of tactile and movement data as a basis for humanoid control, in: Proceedings of the IEEE/RSJ International Conference on Intelligent Robots and Systems, IROS'09, 2009.

[52] T. Takeda, Y. Hirata, K. Kosuge, HMM-based error recovery of dance step selection for dance partner robot, in: Proceedings of the IEEE International Conference on Robotics and Automation, ICRA'07, 2007.

[53] T. Takeda, Y. Hirata, K. Kosuge, Dance partner robot cooperative motion generation with adjustable length of dance step stride based on physical interaction, in: Proceedings of the IEEE/RSJ International Conference on Intelligent Robots and Systems, IROS'07, 2007.

[54] Y. Hirata, S. Komatsuda, K. Kosuge, Fall prevention control of passive intelligent walker based on human model, in: Proceedings of the IEEE/RSJ International Conference on Intelligent Robots and Systems, IROS'08, 2008.

[55] F. DallaLibera, T. Minato, I. Fasel, H. Ishiguro, E. Pagello, E. Menegatti, A new paradigm of humanoid robot motion programming based on touch interpretation, Robotics and Autonomous Systems 57 (8) (2009).

[56] S. Ikemoto, H. B. Amor, T. Minato, H. Ishiguro, B. Jung, Physical interaction learning: behavior adaptation in cooperative human-robot tasks involving physical contact, in: IEEE International Symposium on Robot and Human Interactive Communication, RO-MAN'09, 2009.

[57] R.S. Sutton, A.G. Barto, Reinforcement Learning: An Introduction, The MIT Press, Cambridge, MA, London, England, 1998.

[58] J. Novak, J. Feddema, A capacitance-based proximity sensor for whole arm obstacle avoidance, in: Proceedings of the IEEE International Conference on Robotics and Automation, ICRA'95, 1995.

[59] D. Um, B. Stankovic, E. Giles, T. Hammond, V. Lumelsky, A modularized sensitive skin for motion planning in uncertain environments, in: Proceedings of the IEEE International Conference on Robotics and Automation, ICRA'98, 1998.

[60] H. Cramer, N. Kemper, A. Amin, V. Evers, The effects of robot touch and proactive behaviour on perceptions of human-robot interactions, in: Fourth Annual Conference on Human-Robot Interactions, HRI'09. Short Paper, 2009.

[61] M. Benali-Khoudja, M. Hafez, J.-M. Alexandre, A. Kheddar, Tactile interfaces: a state-of-the-art survey, in: 35th International Symposium on Robotics, ISR'04, 2004.

[62] A. Gallace, H.Z. Tan, C. Spence, The body surface as a communication system: the state of the art after 50 years, in: Presence, MIT Press, Cambridge, MA, USA, 2007, pp. 655-676.

[63] M. Diftler, R. Ambrose, K. Tyree, S. Goza, E. Huber, A mobile autonomous humanoid assistant, in: Proceedings of the IEEE-RAS International Conference on Humanoids Robots, HUMANOIDS'04, 2004.

[64] W. Bluethmann, R. Ambrose, M. Diftler, E. Huber, A. Fagg, M. Rosenstein, R. Platt, R. Grupen, C. Breazeal, A. Brooks, A. Lockerd, R. Peters, O. Jenkins, M. Mataric, M. Bugajska, Building an autonomous humanoid tool user, in: Proceedings of the IEEE-RAS International Conference on Humanoids Robots, 2004.

[65] P.K. Pook, D.H. Ballard, Recognizing teleoperated manipulations, in: Proceedings of the IEEE International Conference on Robotics and Automation, ICRA'93, 1993. 
[66] M. Fujita, H. Kitano, Development of an autonomous quadruped robot for robot entertainment, Autonomous Robots 5 (1) (1998) 7-18.

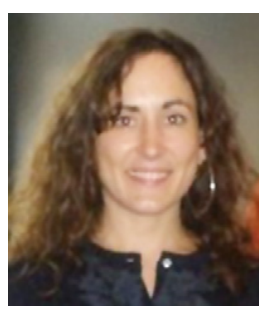

Brenna D. Argall is a postdoctoral fellow in the Learning Algorithms and Systems Laboratory (LASA) at the Swiss Federal Institute of Technology in Lausanne (EPFL). She received her Ph.D. in Robotics (2009) from the Robotics Institute at Carnegie Mellon University, as well as her M.S. in Robotics (2006) and B.S. in Mathematics (2002) Prior to graduate school, she held a Computational Biology position in the Laboratory of Brain and Cognition at the National Institutes of Health, while investigating visualization techniques for neural fMRI data. Her research interests focus upon machine learning techniques to develop and improve robot control systems, under the guidance of a human teacher.

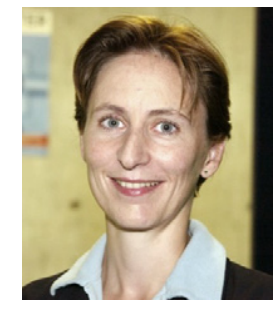

Aude G. Billard is Associate Professor and head of the LASA Laboratory at the School of Engineering at the Swiss Federal Institute of Technology in Lausanne (EPFL).

Prior to this, she was Research Assistant Professor at the Department of Computer Sciences at the University of Southern California, where she retains an adjunct faculty position to this day. Aude Billard received a B.Sc. (1994) and M.Sc. (1995) in Physics from EPFL, with specialization in Particle Physics at the European Center for Nuclear Research (CERN), an M.Sc. in Knowledgebased Systems (1996) and a Ph.D. in Artificial Intelligence (1998) from the Department of Artificial Intelligence at the University of Edinburgh. Her research interests focus on machine learning tools to support robot learning through human guidance. This extends also to research on complementary topics, including machine vision and its use in human-machine interaction and computational neuroscience to develop models of learning in humans. 